\title{
Required role of focal adhesion kinase (FAK) for integrin-stimulated cell
}

\section{migration}

\author{
David J. Sieg*, Christof R. Hauck* and David D. Schlaepfer‡ \\ The Scripps Research Institute, Department of Immunology, IMM26, 10550 N. Torrey Pines Road, La Jolla, CA 92037, USA \\ *These authors contributed equally to this work \\ ¥Author for correspondence (e-mail: dschlaep@scripps.edu) \\ Accepted 24 May; published on WWW 21 July 1999
}

\section{SUMMARY}

FAK localizes to sites of transmembrane integrin receptor clustering and facilitates intracellular signaling events. FAK-null (FAK $\left.{ }^{-}\right)$fibroblasts exhibit a rounded morphology, defects in cell migration, and an elevated number of cell-substratum contact sites. Here we show that stable re-expression of epitope-tagged FAK reversed the morphological defects of the $\mathrm{FAK}^{-}$cells through the dynamic regulation of actin structures and focal contact sites in fibronectin (FN) stimulated cells. FAK re-expressing fibroblasts (clones DA2 and DP3) exhibit a characteristic fibrillar shape and display indistinguishable FN receptorstimulated migration properties compared to normal fibroblasts. Expression of various FAK mutants in the FAK $^{-}$cells showed that FAK kinase activity, the Tyr397/SH2 domain binding site, and the first proline-rich SH3 binding region in the FAK C-terminal domain were individually needed to promote full FAK-mediated FAK ${ }^{-}$ cell migration to $\mathrm{FN}$ whereas direct paxillin binding to FAK was not required. Expression of the FAK Phe-397 mutant did not promote $\mathrm{FAK}^{-}$cell migration and overexpression of $\mathbf{p 5 0}^{\mathrm{csk}}$ in $\mathrm{DA} 2$ cells inhibited migration to FN suggesting that Src-family PTKs play important roles in FAK-mediated motility events. Expression of the FAK C-terminal domain, FRNK, promoted FAK dephosphorylation at Tyr-397 and potently blocked FAKmediated cell migration. This dominant-negative effect of FRNK was reversed by a point mutation (Leu-1034 to Ser) which prevented FRNK localization to focal contact sites. Our results show that FAK functions as a key regulator of fibronectin receptor stimulated cell migration events through the recruitment of both $\mathrm{SH} 2$ and $\mathrm{SH} 3$ domaincontaining signaling proteins to sites of integrin receptor clustering.

Key words: FAK, Fibronectin, Cell migration, Signaling

\section{INTRODUCTION}

FAK was originally identified as a non-receptor proteintyrosine kinase (PTK) localized to focal contact structures. FAK functions as part of a cytoskeletally-associated network of intracellular signaling proteins that are activated by transmembrane integrin receptor clustering (reviewed by Hanks and Polte, 1997). FAK has been shown to facilitate the generation of integrin-stimulated signals to downstream targets such as the ERK2 and JNK/mitogen-activated protein kinase cascades (reviewed by Schlaepfer and Hunter, 1998), to transduce survival signals generated by integrins in adherent cells (Frisch et al., 1996; Ilic et al., 1998), and to play a role in the regulation of cell cycle progression (Zhao et al., 1998).

Genetic support for the role of FAK in integrin-stimulated signaling events comes from results showing that either homozygous deletion of the murine fibronectin (George et al., 1993), $\beta 1$ integrin (Stephens et al., 1995), or FAK (Ilic et al., 1995) genes result in similar early embryonic lethal phenotypes. Comparable abortive gastrulation events (E8.5) due to defective mesodermal development were observed in both the FAK and fibronectin-null mice. Propagation of FAKnull fibroblasts $\left(\mathrm{FAK}^{-}\right)$from E8.0 embryos has been accomplished and these cells exhibit migration but not proliferative defects in cell culture (Ilic et al., 1995). The expression of the FAK-related PTK, Pyk2, is elevated in the FAK $^{-}$cells (Sieg et al., 1998) and normal to elevated tyrosine phosphorylation levels of integrin-associated proteins such as p130 Cas (Vuori et al., 1996), paxillin, and cortactin are found in the FAK $^{-}$cells (Ilic et al., 1995). Although Pyk2 functions in combination with Src-family PTKs to facilitate fibronectinreceptor stimulated Shc tyrosine phosphorylation and ERK2 activation in the $\mathrm{FAK}^{-}$cells, transient Pyk2 overexpression did not reverse the migration defects of these cells whereas transient FAK expression promoted $\mathrm{FAK}^{-}$cell motility (Sieg et al., 1998).

Stable FAK overexpression in Chinese hamster ovary (CHO) cells has been shown to cause enhanced cell migration (Cary et al., 1996, 1998). Both expression of a dominant-negative mutant of FAK termed FRNK (for FAK-related non-kinase domain) (Gilmore and Romer, 1996; Richardson and Parsons, 1996) and studies showing strong correlations between 
elevated FAK expression and the increased invasive potential of human tumors (Owens et al., 1995) have provided support for a role for FAK in cell migration events. In either growing, integrin-stimulated, or in migrating cells, FAK is highly tyrosine-phosphorylated at a number of different sites in vivo (Calalb et al., 1995; Schlaepfer and Hunter, 1996). These phosphorylation events are important for the ability of FAK to promote migration since overexpression of the protein-tyrosine phosphatase (PTP) PTEN has been shown to directly dephosphorylate FAK and antagonize FAK-enhanced cell migration events (Tamura et al., 1998, 1999). However, cells containing an N-terminally truncated form of the Shp-2 PTP (Yu et al., 1998) or fibroblasts deficient in PTP-PEST (AngersLoustau et al., 1999) exhibit elevated FAK tyrosine phosphorylation levels and in addition possess reduced migration capabilities. Therefore, it is believed that repeated cycles of phosphorylation and dephosphorylation may be important factors linking FAK to the promotion of cell motility (reviewed by Schlaepfer et al., 1999).

FAK binds to a number of different signaling proteins via Src homology 2 (SH2) and $\mathrm{SH} 3$ recognition sites and FAKmediated connections with proteins such as paxillin (Richardson et al., 1997), Src-family PTKs (Cary et al., 1996; Fincham and Frame, 1998), p130 Cas (Cary et al., 1998; Klemke et al., 1998), Shc (Schlaepfer et al., 1998), the p85 subunit of phosphatidylinositol 3-kinase (Chen et al., 1996), or the Rho GTPase activating protein GRAF (Hildebrand et al., 1996) could link FAK to pro-migratory signaling pathways. However, no clear model has emerged on how FAK functions in combination with these signaling proteins to promote cell migration. The inability of the $\mathrm{FAK}^{-}$cells to efficiently migrate when presented with a fibronectin $(\mathrm{FN})$ stimulus is believed to be a result of the elevated number and the increased stability of cell-substratum contacts that the $\mathrm{FAK}^{-}$cells make in culture (Ilic et al., 1995). Here we present results of the stable reexpression of FAK within the $\mathrm{FAK}^{-}$cells and the rescue of the morphological and integrin-stimulated migration defects of these cells. Our results support a model whereby FAK is involved in the dynamic regulation of actin and focal contact structures and that FAK functions as an integrin-activated 'scaffold' for the recruitment of both $\mathrm{SH} 2$ and $\mathrm{SH} 3$ domaincontaining signaling proteins needed for $\mathrm{FN}$-stimulated fibroblast cell migration events.

\section{MATERIALS AND METHODS}

\section{Cells}

Primary mouse fibroblasts were isolated from embryonic day $8 \mathrm{fak}^{+/+}$ or $\mathrm{fak}^{-/-}$embryos as described (Ilic et al., 1995). Both the normal $\mathrm{FAK}^{+/+}$and $\mathrm{FAK}^{-/-}$fibroblasts carry mutations in the p53 gene introduced by crossing mice heterozygous for the FAK and p53 alleles as described (Furuta et al., 1995). Clonal populations of early passage $\mathrm{FAK}^{-1-}$ or $\mathrm{FAK}^{+/+}$fibroblasts were isolated by dilution plating. $\mathrm{FAK}^{-1-}$ clones that grew out of this selection exhibited a rounded morphology as initially characterized for the primary $\mathrm{FAK}^{-1-}$ cells (Ilic et al., 1995) whereas $\mathrm{FAK}^{+/+}$clones maintained fibrillar shape. The R6 FAK ${ }^{+/+}$clone was used as the normal fibroblast control and all cells were maintained on gelatin-coated $(0.1 \%$ in phosphatebuffered saline, PBS) cell culture dishes in DMEM supplemented with $10 \%$ fetal bovine serum (FBS), non-essential amino acids for MEM, sodium pyruvate $(1 \mathrm{mM})$, penicillin $(50 \mathrm{U} / \mathrm{ml})$, streptomycin
(50 $\mu \mathrm{g} / \mathrm{ml})$, ciprofloxacin $(0.02 \mathrm{mg} / \mathrm{ml})$, and G418 $(0.5 \mathrm{mg} / \mathrm{ml})$. The $\mathrm{FAK}^{-1}$ clone D cells were transfected with either pcDNA3.1 HAtagged FAK (Sieg et al., 1998) or the pcDNA3.1 empty vector and selected for growth in hygromycin $(200 \mu \mathrm{g} / \mathrm{ml})$. A pooled population of hygromycin-resistant $\mathrm{FAK}^{-/-}$cells was used as the $\mathrm{FAK}^{-}$controls. FAK re-expressing hygromycin-resistant cell clones were isolated by light scatter FACS sorting of single cells into 96-well culture dishes. The 293T human kidney epithelial cells were maintained as previously described (Schlaepfer and Hunter, 1997).

\section{Mutagenesis}

FAK Ser-1034 site-directed mutagenesis was performed using the QuickChange protocol (Clonetech, La Jolla, CA) with a 5'GCTGTGGATGCCAAGAATAGTCTCGATGTTATTGATCAAGCA AG-3' sense primer (antisense not shown) and HA-FAK cloned into pBluescript. Mutants were selected by BsmAI digestion and the integrity of the region was confirmed by DNA sequencing. The cDNA sequence for Ser-1034 FAK was cloned into pcDNA3.1 as a $3.5 \mathrm{~kb}$ BamHI/XbaI fragment whereas Ser-1034 FRNK was cloned into pcDNA3.1 as a $1.4 \mathrm{~kb} A f \mathrm{II} / \mathrm{XbaI}$ fragment. FRNK translation is presumed to start at the Met-691 equivalent site in FAK.

\section{Cell transfection}

Triple HA-tagged Phe-397, Arg-454, Ala-712/713 FAK or triple HAtagged FRNK in pcDNA3 were constructed and used as described (Ilic et al., 1998; Schlaepfer and Hunter, 1996). The pSLX expression vectors for $\mathrm{p} 50^{\text {csk }} \mathrm{WT}$ and kinase-inactive $\mathrm{p} 50^{\text {csk }}(\mathrm{K} 222 \mathrm{M})$ were used as described (Sieg et al., 1998). Transient transfections of FAK ${ }^{-}$cells (primary passages 8-20) were performed using Lipofectamine Plus (Gibco-BRL, Gaithersberg, MD) as per the manufacturer's instructions. Co-transfection of cells with the addition of pcDNA3LacZ followed by staining for $\beta$-gal activity (after 36 hours) using Xgal as a substrate revealed transfection efficiencies at 30 to $40 \%$ for both the $\mathrm{FAK}^{-}$and $\mathrm{FAK}^{+}$cells. Human $293 \mathrm{~T}$ cells were transfected by standard calcium phosphate precipitation techniques as described (Schlaepfer and Hunter, 1997). Trypan blue exclusion assays demonstrated that all transfected cells utilized were $>95 \%$ viable.

\section{Antibodies}

The clone 12CA5 monoclonal antibody (mAb) to the HA-tag was a generous gift from Jill Meisenhelder (The Salk Institute) and the HAtag mAb (clone 16B12) was purchased from Babco (Berkeley, CA). Affinity-purified phosphotyrosine-specific polyclonal antibodies to the motifs surrounding the FAK Tyr-397 site (pY397) or the FAK Tyr577 site (pY577) were kindly provided by Erik Schaefer (QCB Inc., Hopkinton, MA). Anti-P.Tyr (clone 4G10) mAb was purchased from Upstate Biotechnology (Lake Placid, NY) and mAbs to vinculin (clone hVIN-1) and talin (clone 8D4) were purchased from Sigma (St Louis, MO). Monoclonal antibodies to either paxillin (clone 349) or p130 Cas (clone 21) were purchased from Transduction Laboratories (Lexington, KY). Affinity-purified polyclonal antibodies to the FAK N-terminal region (A17) were purchased from Santa Cruz Biotechnology (Santa Cruz, CA) and affinity-purified polyclonal antibodies (\#5904) to FAK residues 8 through 27 were produced as described (Sieg et al., 1998).

\section{Cell stimulation with FN or adherence to poly-L-lysine}

Replating assays were performed on serum-starved (0.5\% FBS for 18 hours) cells harvested by limited trypsin/EDTA treatment as previously described (Sieg et al., 1998). Cells were held in suspension for 1 hour in DMEM containing 0.1\% BSA and then plated onto either FN $(10 \mu \mathrm{g} / \mathrm{ml})$ or poly-L-lysine $(100 \mu \mathrm{g} / \mathrm{ml})$ coated plates for 20 minutes prior to cell lysis.

\section{Cell lysis, immunoprecipitation, and immunoblotting}

Unless otherwise indicated, cell lysates were made in modified RIPA buffer containing $1 \%$ Triton $\mathrm{X}-100,1 \%$ sodium deoxycholate, and 
0.1\% SDS as described (Schlaepfer and Hunter, 1996). Antibodies were incubated in the lysates for 4 hours at $4^{\circ} \mathrm{C}$, and either collected on Protein A (Repligen, Cambridge, MA) or Protein G-plus (Calbiochem, La Jolla, CA) agarose beads. The precipitated protein complexes were washed at $4^{\circ} \mathrm{C}$ in Triton-only lysis buffer (modified RIPA without sodium deoxycholate and SDS) followed by washing in HNTG buffer (50 mM Hepes, $\mathrm{pH} 7.4,150 \mathrm{mM} \mathrm{NaCl}, 0.1 \%$ Triton $\mathrm{X}-100,10 \%$ glycerol) prior to direct analysis by SDS-PAGE. For immunoblotting, proteins were transferred to polyvinylidene fluoride (PVDF) membranes (Millipore, Bedford, MA). Blots were incubated with either $1 \mu \mathrm{g} / \mathrm{ml}$ monoclonal, 1:1000 dilution of polyclonal antibodies, or with FAK phosphotyrosine-specific antibodies at 0.5 $\mu \mathrm{g} / \mathrm{ml}$ for 2 to 4 hours at RT. Bound primary antibody was visualized by enhanced chemiluminescent detection and subsequent reprobing of membranes was performed as described (Schlaepfer et al., 1998).

\section{Immunofluorescence staining}

Serum-starved cells were either plated (DMEM with $0.1 \%$ BSA) onto FN-coated $(10 \mu \mathrm{g} / \mathrm{ml})$ glass coverslips for 2 hours or grown (DMEM containing $5 \% \mathrm{FBS}$ ) on $\mathrm{FN}$-coated coverslips overnight prior to fixation with $3.7 \%$ paraformaldehyde. Samples were permeabilized with ice-cold acetone for 10 minutes, washed in PBS, and blocked with the avidin-biotin-kit (Vector, Burlingame, CA) for HA-tagstaining (clone 16B12) or for 10 minutes with blocking buffer (PBS, $0.5 \%$ BSA, $200 \mu \mathrm{g} / \mathrm{ml}$ ChromaPure goat IgG; Jackson ImmunoResearch Laboratories, West Grove, PA) for vinculin, paxillin, and talin staining. Primary antibodies were diluted in blocking buffer and applied for 1 hour at RT. After three washes in PBS, coverslips were incubated for 45 minutes in blocking buffer containing either biotin-conjugated horse anti-mouse (Vector) for HAtag staining or FITC-conjugated goat anti-mouse antibodies (Jackson). In some cases, actin was visualized by the addition of rhodamine-phalloidin (Molecular Probes, Eugene, OR). After washing, vinculin/paxillin/talin stained samples were mounted in Vectashield (Vector), whereas HA-tag stained samples were incubated for 30 minutes with FITC-avidin (Vector) before washing and mounting. Control stainings were performed without either primary or secondary antibodies. Samples were viewed with an Olympus BX60 epifluorescence microscope equipped with appropriate filters and images were photographed with Kodak TMAX 400 film, scanned, and reconstructed using Adobe PhotoShop software.

\section{Wound healing assay}

Exponentially growing cells $\left(2 \times 10^{6}\right)$ were plated onto rat tail collagen (Boerhinger Mannheim, Indianapolis, IN) coated $(10 \mu \mathrm{g} / \mathrm{ml})$ cell culture plates in complete growth media. After 8 hours, the monolayer of cells were wounded by manual scratching with a pipet tip, washed with PBS, photographed in phase contrast with a Nikon microscope ( 0 hour point) and placed into complete growth medium. Matched pair marked wound regions were photographed again after 18 hours.

\section{Cell migration assays}

The MilliCell modified Boyden chamber (Millipore, Bedford, MA) migration assays were performed as previously described (Sieg et al., 1998). Cells were used 36 hours after transfection with the indicated constructs following overnight serum starvation (0.5\% FBS). Chambers for haptotaxis assays were prepared by pre-coating the under surface of the polycarbonate membrane with FN $(10 \mu \mathrm{g} / \mathrm{ml}$ in PBS) for 2 hours at $37^{\circ} \mathrm{C}$ during which time the upper chamber contained $0.05 \%$ BSA in DMEM. To initiate migration assays, chambers were washed in PBS and cells $\left(1 \times 10^{5}\right.$ cells in $0.3 \mathrm{ml}$ DMEM with $0.5 \% \mathrm{BSA}$ ) were added to the upper chamber. After 3 hours at $37^{\circ} \mathrm{C}$ in the absence of serum, the cells on the upper surface of the membrane were removed by a cotton tip applicator and the migratory cells on the lower membrane surface were fixed by methanol/acid treatment and stained with Crystal Violet $(0.1 \%$ Crystal Violet, $0.1 \mathrm{M}$ borate $\mathrm{pH} 9.0,2 \% \mathrm{EtOH})$ or analyzed for $\beta$-galactosidase $(\beta$-gal) activity using $\mathrm{X}$-gal as a substrate. Cell migration values were determined either by elution of the Crystal Violet stain in $10 \%$ acetic acid and measuring absorbance at $600 \mathrm{nM}$ or by counting $\beta$-gal stained cells (cells/field using a $\times 40$ objective). Each determination represents the average of three individual wells and error bars represent standard deviation (s.d.). By cell counting methods, background levels of cell migration to $0.5 \% \mathrm{BSA}$ were $<1 \%$ of values obtained with FN. Data presented are representative of at least three separate experiments.

\section{Statistical analysis}

Ordinary one-way analysis of variance (ANOVA) was used to determine the overall significance within data groups. If a significant result was obtained by ANOVA, the Tukey-Kramer multiple comparisons $t$-test was used to determine significance between individual groups.

\section{RESULTS}

\section{Stable re-expression of epitope-tagged FAK in FAK- cells re-establishes wild-type fibroblast phenotype}

FAK $^{-}$cells readily proliferate in culture and they exhibit an elevated expression of the FAK-related Pyk2 PTK compared to normal fibroblasts. In contrast to the focal contact localization of FAK, Pyk2 exhibits a peri-nuclear distribution in the $\mathrm{FAK}^{-}$cells (Sieg et al., 1998). Transient overexpression of Pyk2 only weakly enhanced $\mathrm{FAK}^{-}$cell migration whereas transient FAK expression promoted $\mathrm{FAK}^{-}$cell migration to FN equal to normal fibroblasts (Sieg et al., 1998). To test the hypothesis that FAK and Pyk2 have distinct and nonoverlapping functional properties, hygromycin drug selection was used to facilitate the stable re-expression of FAK. The FAK ${ }^{-}$cells used for these studies were early passage cells clonally selected for rapid growth and displayed a highly rounded phenotype. Hygromycin resistant pools of FAKtransfected cells exhibited low levels of FAK expression and displayed both rounded and spread morphologies (data not shown). After further passage and expansion of this cell pool, only rounded cells were observed. At the same time, detectable FAK re-expression was lost from the pooled population. Therefore, FAK re-expressing cells were obtained by clonal selection and expansion from the initial hygromycin resistant cell pool (Fig. 1). Clonal-derived FAK re-expressing cells grew slower than the hygromycin empty vector transfected control FAK $^{-}$cells (D. Sieg and D. Schlaepfer, unpublished results) and all identified FAK re-expressing cells exhibited a morphological reversion to a wild-type spread fibroblast phenotype (see Fig. 1C). No significant changes in the level of Pyk2 mRNA expression were detected in the FAK reexpressing cells compared to the $\mathrm{FAK}^{-}$cell clone used in these analyses (data not shown).

No clones were obtained that overexpressed FAK compared to normal fibroblasts $\left(\mathrm{FAK}^{+/+}\right)$. Instead, FAK re-expression was $\sim 50$ to $75 \%$ of the level of endogenous FAK in normal fibroblasts (Fig. 1A). The FAK construct used for re-expression in the $\mathrm{FAK}^{-}$cells contained a triple $\mathrm{C}$-terminal hemagglutinin (HA) epitope-tag and identification of HA-tagged FAK which exhibited reduced mobility after SDS-PAGE $(\sim 120 \mathrm{kDa})$ was performed by immunoblotting with antibodies (12CA5) to the HA-epitope tag (Fig. 1A). Since it is possible that the addition of this HA-tag to FAK could influence its activity, standard FN 
A
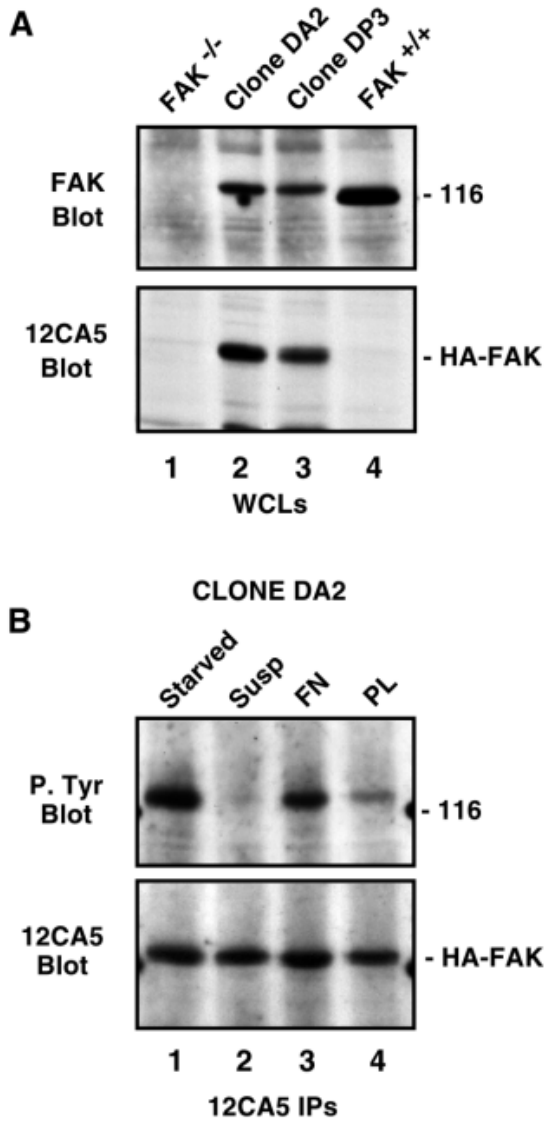

C

\section{Subconfluent}

$(8 \mathrm{~h})$
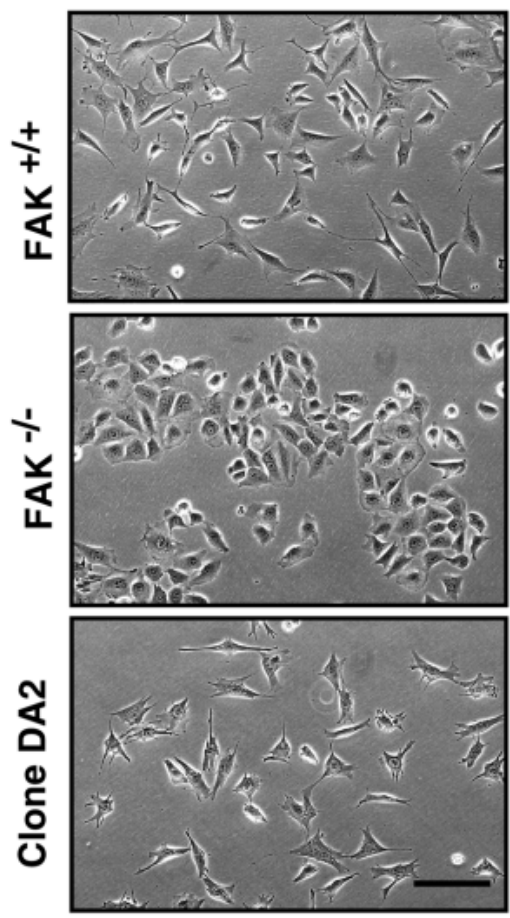

Confluent

(96 h)

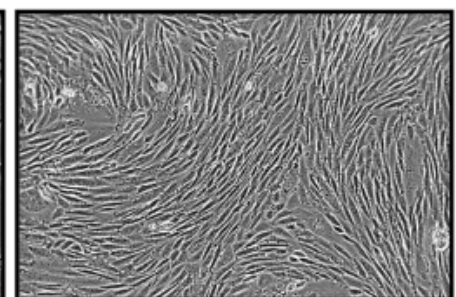

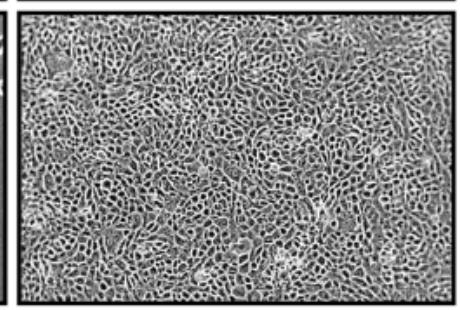

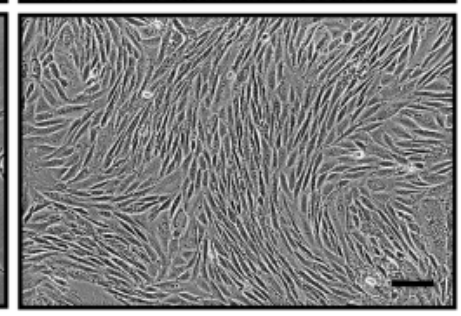

Fig. 1. Stable FAK re-expression promotes $\mathrm{FAK}^{-1-}$ fibroblast morphology changes. (A) Whole cell lysates were prepared and $100 \mu \mathrm{g}$ total cell

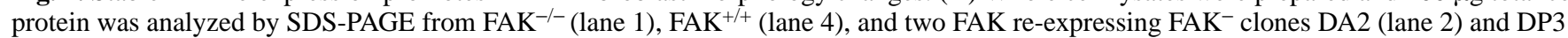
(lane 3). FAK protein expression was analyzed by blotting with polyclonal anti-FAK (A-17) antibodies and HA-tagged FAK expression was detected by 12CA5 mAb blotting. (B) Lysates were prepared from DA2 cells that were either serum-starved (starved, lane 1), held in suspension for 1 hour (Susp, lane 2), and then replated onto FN-coated (FN, lane 3) or poly-L-lysine-coated (PL, lane 4) dishes for 20 minutes. HA-FAK IPs (12CA5 mAb) were analyzed by anti-P.Tyr blotting and then by anti-HA tag blotting of the same membrane. (C) FAK ${ }^{-/-}$, normal $\mathrm{FAK}^{+/+}$, and clone DA2 fibroblasts were plated onto gelatin coated dishes and images were taken by phase contrast microscopy at subconfluent ( 8 hours) and confluent cell densities (96 hours). Bars, $100 \mu \mathrm{m}$.

replating assays were performed with both the DA2 and DP3 clones of the FAK re-expressing cells (Fig. 1B). Cells were either serum starved, held in suspension for 1 hour, or replated onto either FN $(10 \mu \mathrm{g} / \mathrm{ml})$ or poly-L-lysine $(100 \mu \mathrm{g} / \mathrm{ml})$ coated plates for 20 minutes and HA-FAK in cell lysates was analyzed by 12CA5 immunoprecipitation and anti-phosphotyrosine (P.Tyr) blotting (Fig. 1B). HA-FAK was highly tyrosinephosphorylated in serum starved cells, dephosphorylated in suspended cells, and rapidly re-phosphorylated after FN but not poly-lysine replating. This regulation of HA-FAK tyrosine phosphorylation in clones DA2 (Fig. 1B) and DP3 (data not shown) was identical to the regulation of FAK in normal fibroblasts and epithelial cells (Schlaepfer et al., 1998). These results indicate that the addition of a C-terminal epitope tag to FAK did not adversely affect measurable FAK function in the DA2 fibroblasts.

Phase contrast microscopy revealed that the morphology of the DA2 and DP3 FAK re-expressing clones were identical to that of normal fibroblasts (Fig. 1C and data not shown). When grown at low cell densities, DA2 and DP3 fibroblasts proliferated as separate cells whereas the control hygromycinresistant $\mathrm{FAK}^{-}$fibroblasts tended to grow in clusters. At higher cell densities, the DA2 and DP3 cells formed a fibrillar pattern characteristic of normal mouse embryo fibroblasts whereas the $\mathrm{FAK}^{-}$cells formed a tight monolayer of rounded cells (Fig. 1C). These results show that HA-tagged FAK expression in the $\mathrm{FAK}^{-}$cells re-established the wild-type fibroblast phenotype.

\section{FAK promotes the dynamic regulation of actin structures and focal contact sites in FN-stimulated fibroblasts}

When fibroblasts are plated onto extracellular matrix proteins such as FN in the absence of serum co-factors, they undergo rapid cell spreading and assume a polarized morphology within 2 hours. During this early period of rapid cell spreading, FAK exhibits up to a 10-fold elevated level of measurable in vitro kinase activity in NIH3T3 fibroblasts (Schlaepfer et al., 1998). To study these spreading events, normal $\mathrm{FAK}^{+/+}, \mathrm{FAK}^{-}$, and the DA2 fibroblasts were either plated onto FN glass coverslips for up to 2 hours in the absence of serum prior to immunofluorescence (IF) analyses (Fig. 2). A time course with normal fibroblasts plated onto FN for 20 minutes to 2 hours was established by double-labeling for vinculin and actin (Fig. 2A). After 20 minutes, the cells that had started to spread 
A

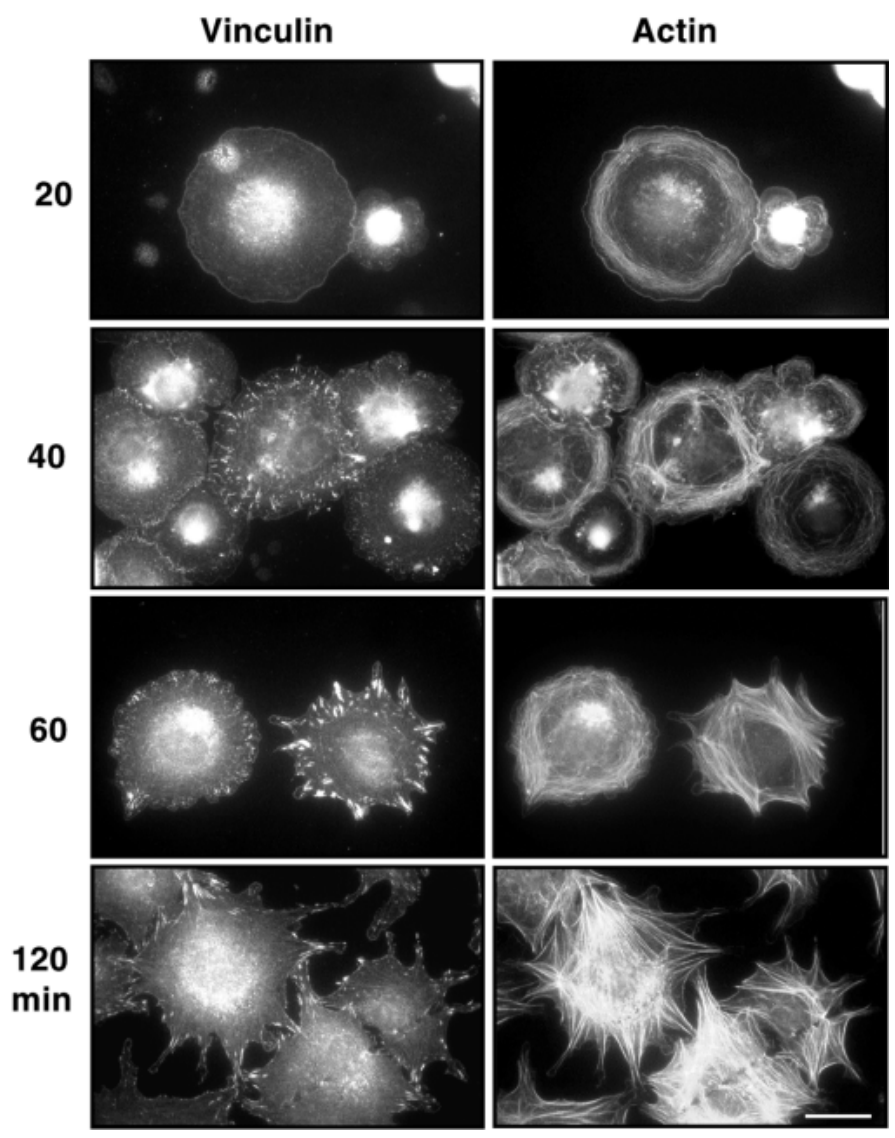

Fig. 2. Remodeling of actin cytoskeletal structures and focal contact sites in spreading cells. (A) $\mathrm{FAK}^{+/+}$fibroblasts were allowed to adhere to a FN-coated surface for the indicated times in the absence of serum and co-stained for vinculin and actin. Images at 20 minutes represent a subpopulation of highly spread cells.

(B) Immunolocalization of FAK, actin and the focal contact associated protein vinculin in $\mathrm{FAK}^{-/-}$and DA2 fibroblasts. $\mathrm{FAK}^{-1-}$ and clone DA2 cells were allowed to attach on a FN-coated surface for 2 hours in the absence of or 16 hours in the presence of serum, fixed, permeabilized and stained for vinculin or the HA-tag with monoclonal antibodies and FITC-coupled secondary reagents and for actin with phalloidin-TRITC. The image of $\mathrm{FAK}^{-1-}$ cells stained with the HA-tag-antibody (16B12) was taken with an extended exposure time to reveal background staining. Bar, $25 \mu \mathrm{m}$. showed prominent vinculin staining in the center of the cells, whereas cortical actin rings were present around the cell periphery. After 40 to 60 minutes patches of vinculin staining appeared at the cell periphery, whereas vinculin staining in the cell center lessened. By 60 minutes, $\sim 50 \%$ of the cells exhibited strong bright patches of vinculin staining in distinct spots at the cell periphery and these sites were associated with the ends of actin stress fibers which had emanated from reorganization of the cortical actin ring structures. The clustered vinculin stained patches most likely represent the formation of focal contact sites. Importantly, it was between 60 minutes and 2 hours that the FN-stimulated $\mathrm{FAK}^{+}$cells exhibited actin stress fiber contractile changes which resulted in a pointed or stellate cell morphology (Fig. 2A).

When replated onto $\mathrm{FN}$-coated slides, the $\mathrm{FAK}^{-}$cells showed similar kinetics of initial cell spreading and actin

\section{B}
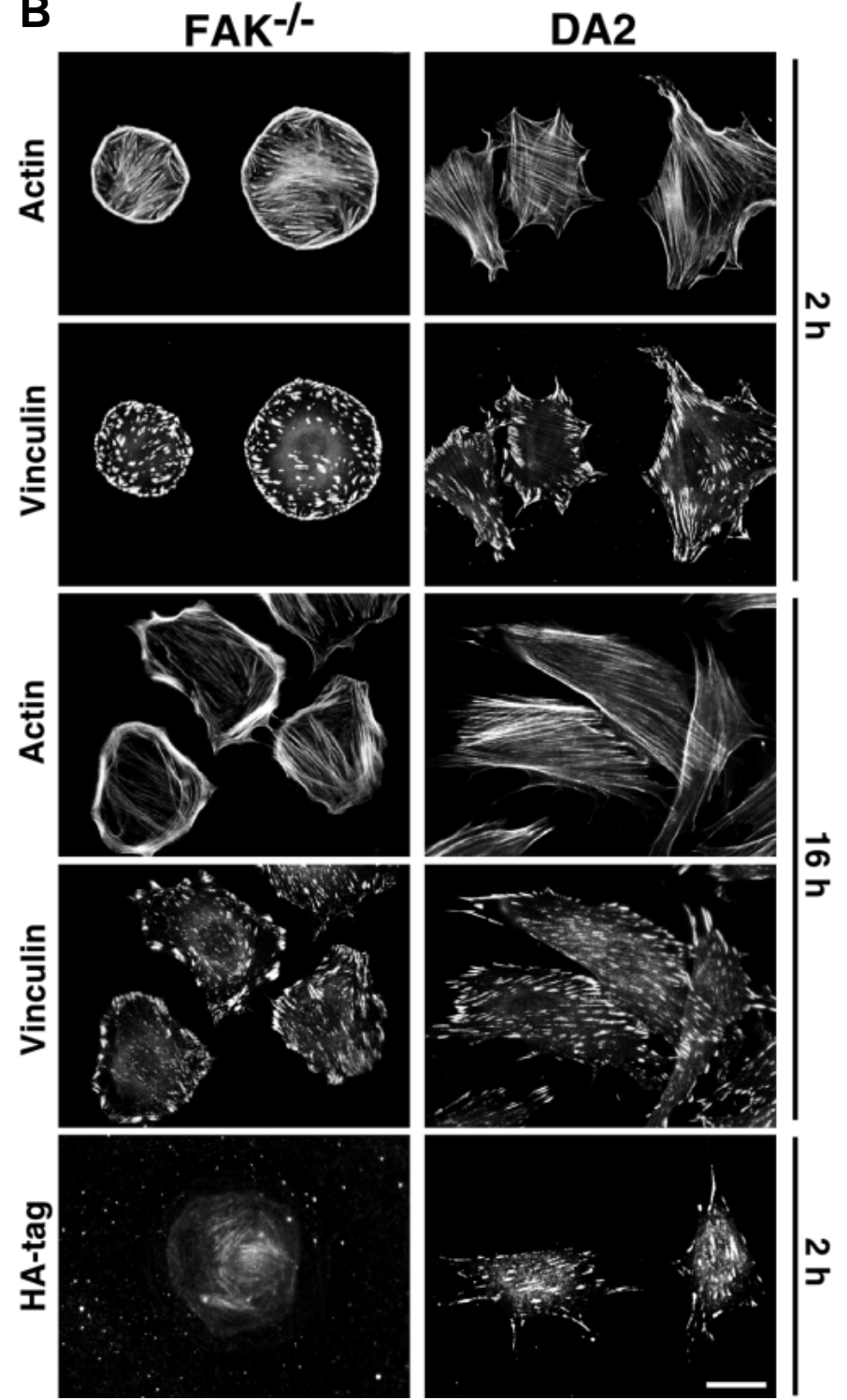

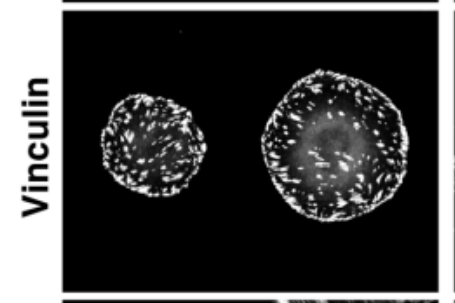

cortical ring formation (data not shown). Significantly, after 2 hours on FN, the cortical actin ring became very dense around the cell periphery and the cells still exhibited an elevated number of small vinculin-stained patches in the center and in the periphery of the rounded $\mathrm{FAK}^{-}$cells (Fig. 2B). Compared to the DA2 fibroblasts which attained a pointed and elongated morphology after 2 hours on FN, the $\mathrm{FAK}^{-}$cells seemed to display reduced actin stress fiber contractility events and remained in a very static and rounded structure. Since spreading could just be delayed in $\mathrm{FAK}^{-}$cells, cells were stained for actin and vinculin that had been plated onto FN for 16 hours in the presence of $0.5 \%$ FBS. After 16 hours, the DA2 cells had spread considerably and occupied a larger surface area than DA2 cells after 2 hours on FN. Actin stress fibers in the DA2 cells were organized in long parallel projections and were equally distributed throughout the cell. In contrast, $\mathrm{FAK}^{-}$ 
cells still displayed a compact shape after 16 hours and filamentous actin was still enriched around the perimeter of the $\mathrm{FAK}^{-}$cells. After extended incubation on FN, vinculin could be found in distinct spots mainly at the cell periphery colocalizing with the tips of actin stress fibers in both DA2 and FAK $^{-}$cells (Fig. 2B). Vinculin positive patches in the DA2 cells appeared extended, but showed no gross difference in their distribution compared to the $\mathrm{FAK}^{-}$cells.

IF localization with antibodies to the HA-tag revealed that FAK staining was most prominent at the perimeter regions and at the ends of actin stress fibers or cell extensions in the DA2 cells (Fig. 2B). This distribution of FAK in the DA2 cells is consistent with the observed focal contact association of FAK in normal fibroblasts (Sieg et al., 1998). In contrast, antibodies to the HAtag did not stain similar structures in $\mathrm{FAK}^{-}$cells (Fig. 2B). These results support the hypothesis that FAK localization to focal contact sites promotes signals leading to actin contractility events and the dynamic regulation of focal contact structures in spreading cells. In the absence of FAK, the cells were unable to readily assume a pointed or elongated morphology.

To determine whether FAK influences cell morphology by altering the distribution of other focal contact-associated proteins, we performed IF analyses for paxillin, talin, and actin distribution

A
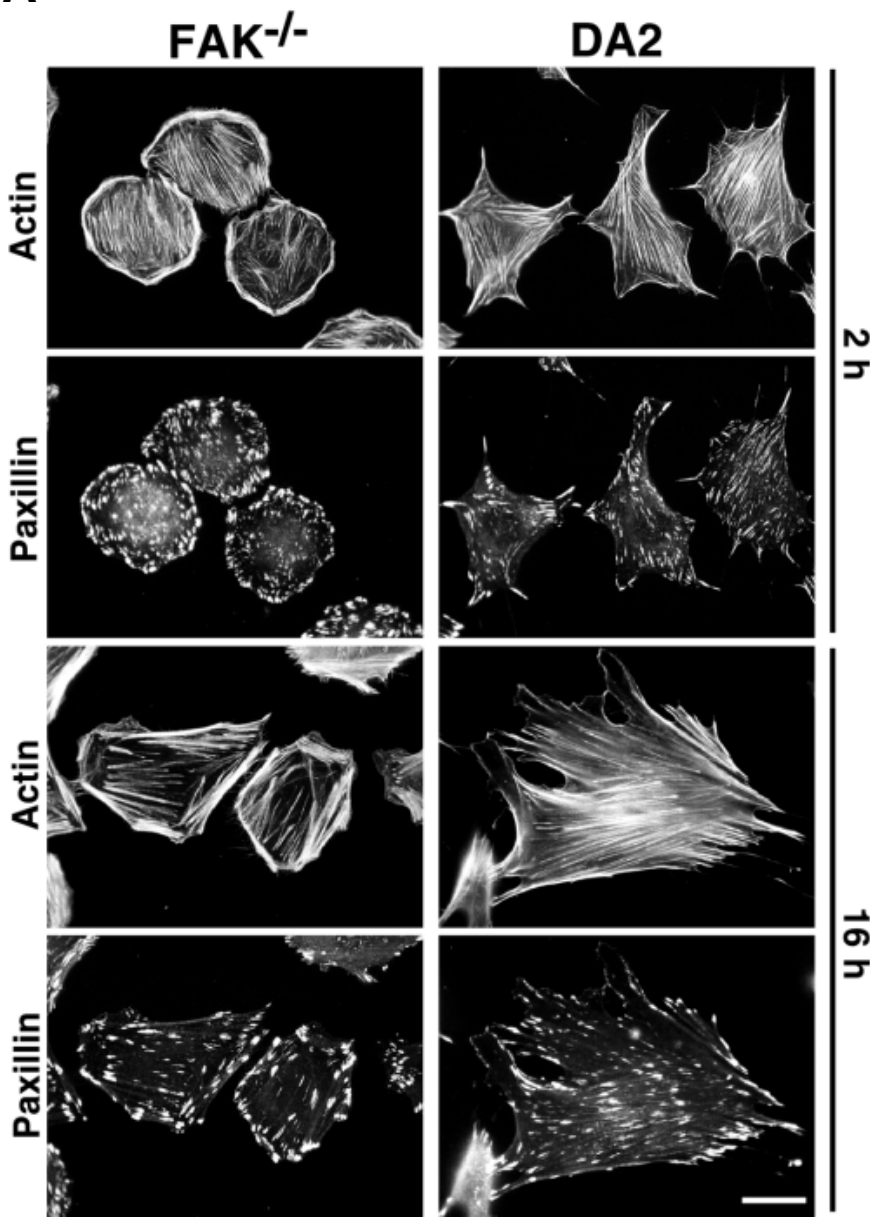

in the DA2 and FAK ${ }^{-}$cells. Again, FAK ${ }^{-}$cells plated for 2 hours onto FN showed a dense circumferential actin ring (Fig. 3A and $\mathrm{B})$, where numerous small focal contact sites had accumulated containing both paxillin (Fig. 3A) and talin (Fig. 3B). In contrast, DA2 cells started to elongate at this early time point with paxillin (Fig. 3A) and especially talin (Fig. 3B) enriched in the outermost focal contacts. After 16 hours on $\mathrm{FN}$ in the presence of serum, DA2 cells attained an extended and pointed cell shape, whereas $\mathrm{FAK}^{-}$cells retained their compact morphology with strong actin staining remaining in the cell periphery (Fig. 3A). Interestingly, both paxillin (Fig. 3A) as well as talin (Fig. 3B) were organized in well defined patches at the end of actin stress fibers in both the $\mathrm{FAK}^{-}$and the DA2 cells. These results show that FAK localization to focal contact sites does not affect the overall distribution of vinculin or the FAK binding proteins paxillin and talin. Instead, FAK appears to influence cell morphology by regulating the turnover rate of focal contact sites and the reorganization of peripheral actin structures.

\section{Re-expression of FAK rescues the FAK- cell migration defect to FN}

Since the DA2 and DP3 FAK re-expressing cells were morphologically identical to normal $\mathrm{FAK}^{+/+}$fibroblasts, wound

B

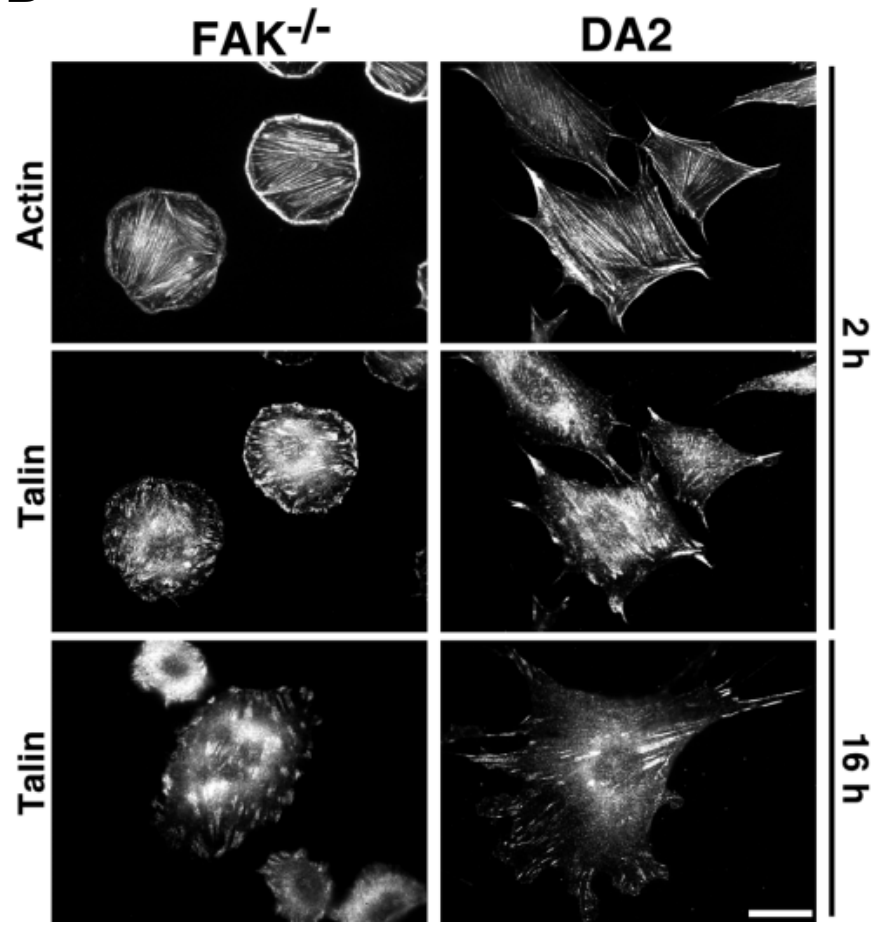

Fig. 3. Paxillin and talin exhibit similar distributions in the $\mathrm{FAK}^{-1-}$ and DA2 cells. $\mathrm{FAK}^{-/-}$and DA2 cells were allowed to attach to a FN-coated surface for 2 hours in the absence of or 16 hours in the presence of serum, fixed, permeabilized and stained for paxillin (A) or talin (B) with monoclonal antibodies and FITC-coupled secondary reagents and for actin with phalloidin-TRITC. Bars, $25 \mu \mathrm{m}$. 
healing scratch assays were performed to determine whether stable HA-FAK expression would also reverse the migration defects of the $\mathrm{FAK}^{-}$cells (Fig. 4A and data not shown). Equal numbers of growing cells $\left(2 \times 10^{6}\right)$ were plated onto $10 \mathrm{~cm}$ dishes and after 8 hours, cells were cleared within a defined area by scratching with a pipet tip, washed with PBS, and allowed to migrate into the cleared area in the presence of serum (Fig. 4A, 0 hours). Eighteen hours after the scratch, the $\mathrm{FAK}^{-}$cells had become quite dense along the wound edge due to continued cell proliferation, however, they did not significantly migrate into the cleared area or show changes in cell morphology (Fig. 4A, 18 hours). Within the same eighteen hour period, cells from both the normal and DA2 fibroblasts had separated from the monolayer at the wound edges and exhibited intense polarized membrane protrusions characteristic of the leading lamellae of migratory cells (Fig. 4A, 18 hours). In addition, cells within the wound edge of the DA2 fibroblasts also exhibited areas where the cells had become reoriented perpendicular to the scratched region. Significantly, the time course of total wound closure (30 hours) was identical for the normal $\mathrm{FAK}^{+/+}$and DA2 fibroblasts whereas wound closure was observed at $\sim 48$ hours for the $\mathrm{FAK}^{-}$cells and was presumed to be due to continued cell proliferation (data not shown).

To measure quantitatively the effect of FAK on FN- stimulated cell migration events, modified Boyden chamber haptotaxis migration assays were performed with FN (10 $\mu \mathrm{g} / \mathrm{ml}$ ) for 3 hours in the absence of serum (Fig. 4B). The $\mathrm{FAK}^{-}$, DA2, DP3, and normal fibroblasts equally adhered to the tissue cultured treated membrane and showed only a low level of random cell migration on BSA-coated membranes (Fig. 4B, BSA control). When FN was used to coat $(10 \mu \mathrm{g} / \mathrm{ml})$ the underside of the Boyden chamber membrane, both the $\mathrm{FAK}^{+/+}, \mathrm{DA} 2$, and DP3 fibroblasts readily migrated to the FN surface underside whereas the $\mathrm{FAK}^{-}$cells did not migrate (Fig. 4B). By cell counting methods, the FN-stimulated level of migration was over 10-fold higher than the BSA controls for both the normal and DA2 fibroblasts (data not shown). Crystal Violet dye elution from the migrating cells yielded increased $\mathrm{FAK}^{+/+}$, DA2, and DP3 migration values 4- to 5-fold higher than controls (Fig. 4B). This fold increase in cell migration obtained for the dye elution method was less than that obtained by cell counting due to non-specific Crystal Violet staining of the BSA-coated control membranes. Importantly, when both sides of the Boyden chamber membrane were coated with FN, neither the normal, DA2, nor DP3 cells migrated to the underside (data not shown). These results show that FAK reexpression did not increase random cell motility. Instead, FAK functions to connect $\mathrm{FN}$ receptor integrins to intracellular signaling events promoting cell migration.
A
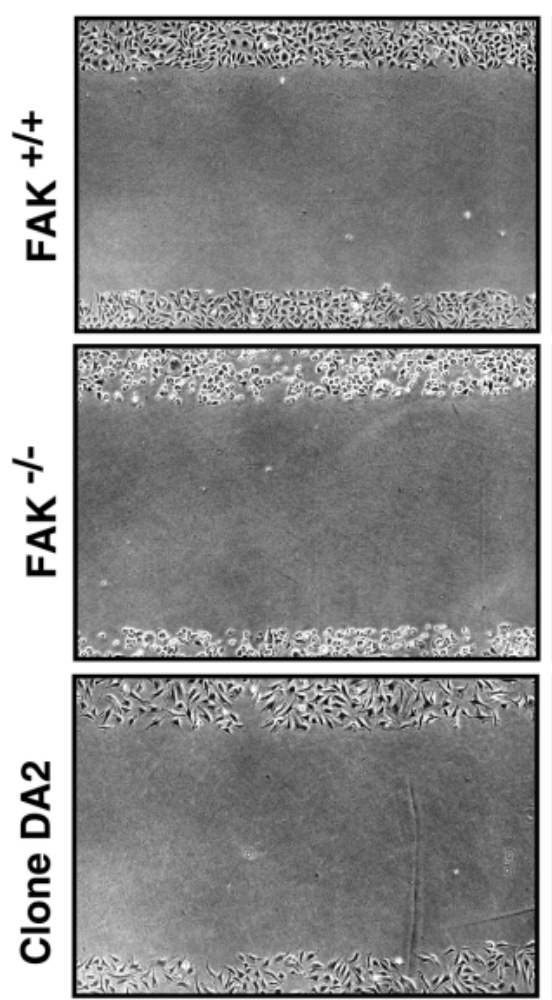

$18 \mathrm{~h}$
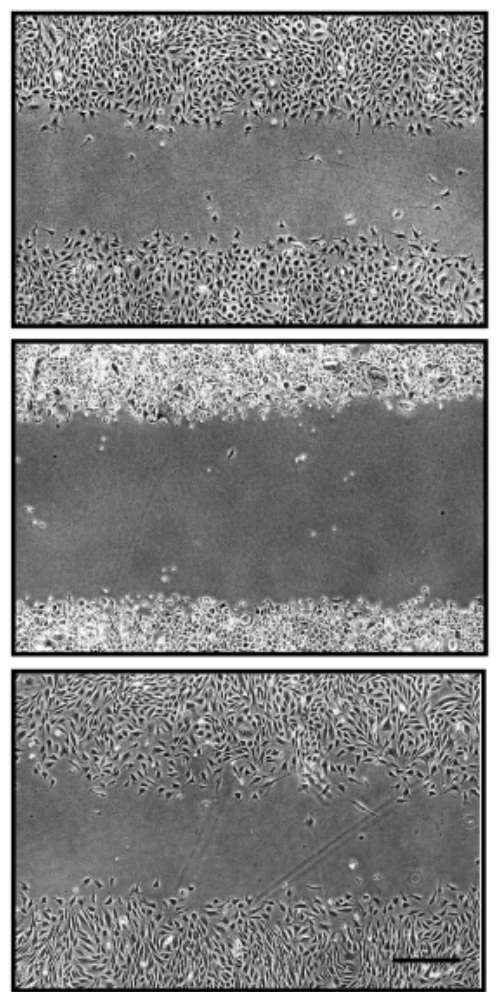

B

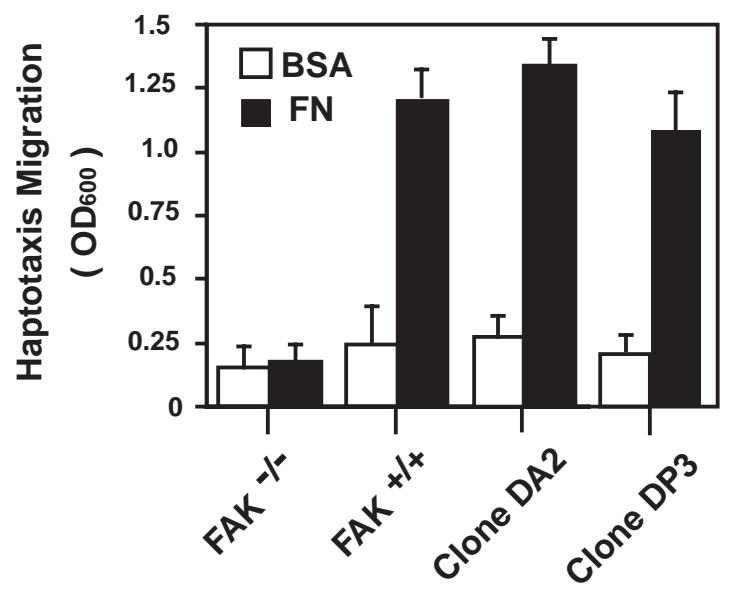

Fig. 4. Stable FAK re-expression rescues $\mathrm{FAK}^{-1-}$ cell migration defects. (A) $\mathrm{FAK}^{+/+}, \mathrm{FAK}^{-1-}$ and DA2 fibroblasts were plated onto $10 \mathrm{~cm}$ dishes and allowed to grow in the presence of $10 \%$ FBS. After 8 hours, a wound was created by scratching with a pipet tip (0 h) and the cells were then allowed to migrate into the wounded area in the presence of $10 \%$ FBS. Phase contrast images were taken 18 hours later to assess cell migration. (B) $\mathrm{FAK}^{+/+}, \mathrm{FAK}^{-/-}$, DA2, and DP3 fibroblasts were allowed to migrate for 3 hours towards fibronectin (FN) or BSA (BSA) immobilized on the undersurface of a modified Boyden chamber in a haptotaxis migration assay performed in the absence of serum. The cells that migrated to the underside of the chamber were stained with Crystal Violet, the dye eluted in $10 \%$ acetic acid, and the absorbance quantified at $600 \mathrm{~nm}$. Shown is the mean \pm standard deviations of triplicates from at least three independent experiments. 
FAK kinase activity, the autophosphorylation site, and the first proline-rich motif are all important for cell migration

To determine the mechanism(s) by which FAK re-expression was overcoming the migration defect in the $\mathrm{FAK}^{-}$ fibroblasts, FN haptotaxis Boyden chamber migration assays were conducted with $\mathrm{FAK}^{-}$cells transiently transfected with various HA-tagged FAK mutant constructs (Fig. 5). To visualize and score only the FAK-transfected cells in this assay, a $\beta$-galactosidase expression vector was cotransfected with the FAK constructs, migratory cells on the membrane underside were stained with X-gal (see Fig. 6B) and only the blue cells were counted (Fig. 5). Wild-type (WT) FAK expression promoted an $\sim 11$-fold increase in FN-stimulated cell migration compared to vector control transfected $\mathrm{FAK}^{-}$cells. Expression of the autophosphorylation and $\mathrm{SH} 2$ domain binding site mutant of FAK (Phe-397) did not significantly increase $\mathrm{FAK}^{-}$cell
A

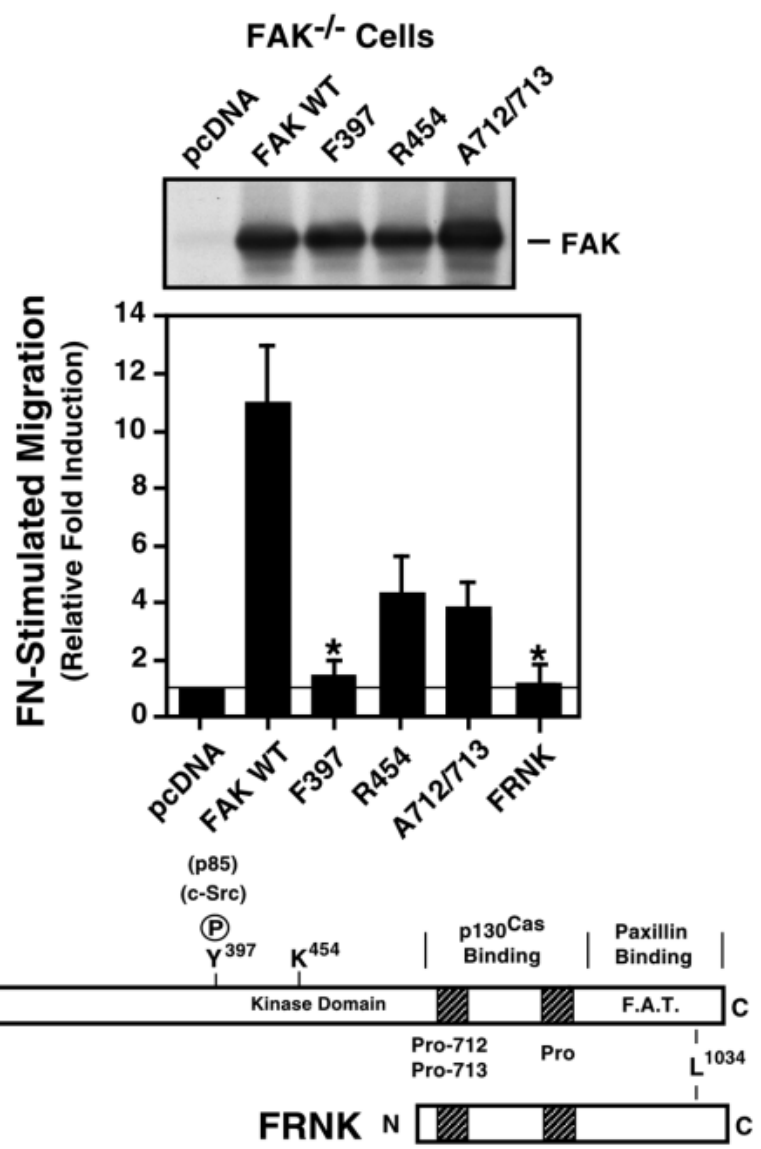

B

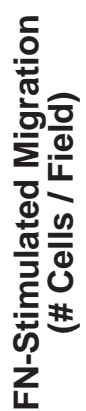

Clone DA2 Cells

C

Transfected FAK $^{-/-}$Cells
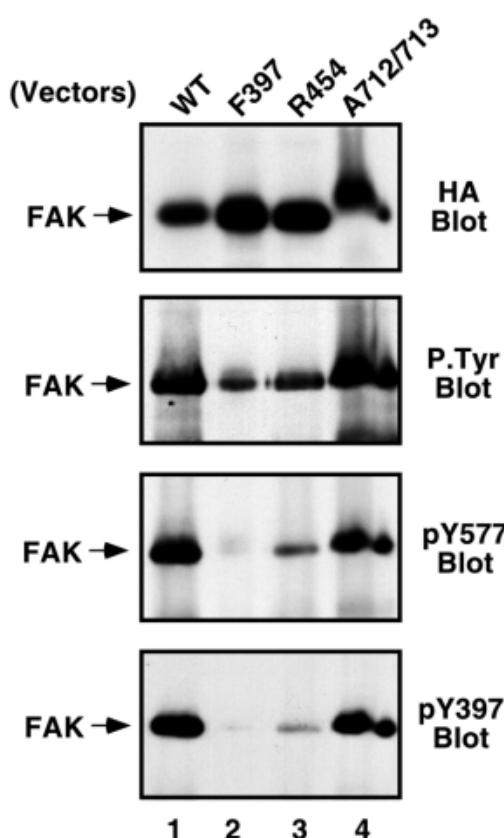

FN-Stimulated

Fig. 5. Multiple protein binding sites on FAK are required to promote cell motility to FN. (A) $\mathrm{FAK}^{-/-}$cells were transiently co-transfected with $\beta$-gal and either wildtype FAK (FAK WT), FAK Phe-397 (F397), kinase-inactive Arg-454 FAK (R454), FAK Ala-712/713 (A712/713), FRNK or the empty vector (pcDNA) and analyzed in a modified Boyden-chamber FN haptotaxis assay. The corresponding amino acid point mutations and corresponding binding domains are depicted in the illustration of the FAK and the C-terminal domain FRNK proteins. Equal amounts of FAK protein expression was verified by HA-tag (12CA5) blotting of whole cell lysates prepared from excess cells not used the migration assay. Cells that migrated to the underside of the migration chamber were stained with X-gal and only the cells that stained blue counted. Relative fold induction was calculated by normalization of the transfection-induced number of migrating cells in each experiment. Each bar represents the mean and standard deviation of triplicates from at least three independent experiments. Migration of cells expressing the mutant FAK constructs was significantly different from FAK WT-induced migration $(P<0.001)$. *Significantly different from R454- and A712/713-induced migration $(P<0.05)$. (B) DA2 cells were transiently co-transfected with $\beta$-gal and either wild-type CSK, kinase-inactive (K222M) CSK or the empty vector (pcDNA) and analyzed in a modified Boyden-chamber FN haptotaxis assay. Each bar represents the mean and standard deviation of triplicates from at least three independent experiments. (C) FAK ${ }^{-1-}$ cells were transiently transfected with either wild-type FAK (FAK WT), FAK Phe-397 (F397), kinase-inactive Arg-454 FAK (R454) or FAK Ala-712/713 (A712/713). These cells were replated onto FN-coated dishes for 1 hour in the absence of serum. HA-tag IPs were analyzed by blotting with anti-HA tag (16B12), anti-P.Tyr (4G10), or phosphotyrosinespecific antibodies to motifs surrounding FAK Tyr-577 (pY577) or FAK Tyr-397 (pY397). 
motility over the low basal level of FN-stimulated $\mathrm{FAK}^{-}$cell migration (Fig. 5A).

Expression of kinase-inactive FAK (Arg-454) weakly promoted $\mathrm{FAK}^{-}$cell migration to $\mathrm{FN}$ at a level 3-fold less than WT FAK (Fig. 5A), which was a level significantly above Phe397 FAK. This result differs from those obtained from FAK overexpression studies in $\mathrm{CHO}$ cells where kinase-inactive FAK equally enhanced FN-stimulated cell migration compared to WT FAK (Cary et al., 1996). Interestingly, expression of FAK mutated at the first proline-rich site (Ala-712/713), a site previously shown to mediate interactions with $\mathrm{SH} 3$ domaincontaining proteins such as p130 Cas (Polte and Hanks, 1995), also only weakly promoted $\mathrm{FAK}^{-}$cell migration to $\mathrm{FN}$ at a level 3-fold less than WT FAK and at a level significantly above Phe-397 FAK (Fig. 5A). Overexpression of a similar Ala-712/715 FAK mutant in CHO cells did not promote enhanced migration (Cary et al., 1998). The fact that the individual Phe-397, Arg-454, or Ala-712/713 FAK mutations prevented full FAK function in promoting $\mathrm{FAK}^{-}$cell migration suggests that FN-stimulated increases in FAK kinase activity trigger an important coordination of both $\mathrm{SH} 2$ and $\mathrm{SH} 3$ domain-containing proteins with FAK. The fact that both Arg454 and Ala-712/713 FAK promoted significantly more cell migration than Phe-397 FAK suggest that the integrity of the FAK autophosphorylation site is of critical importance for cell motility signaling events.

One important class of signaling proteins which interact with FAK at the Tyr-397 site after FN stimulation of cells are the Src-family PTKs. FN stimulation of fibroblasts has been shown to promote the transient association of c-Src with FAK leading to either Src-mediated phosphorylation of other FAK tyrosine residues creating new $\mathrm{SH} 2$ binding sites (Schlaepfer et al., 1994) or Src-mediated phosphorylation of FAK kinase domain residues leading to enhanced FAK catalytic activity (Calalb et al., 1995). To determine the importance of Srcfamily PTK activity in FAK-mediated cell migration to FN, the DA2 cells were transiently-transfected with p50 csk which phosphorylates the C-terminal regulatory site in Src-family PTKs and prevents full Src PTK activity after cell stimulation (Fig. 5B). Compared to the lacZ control vector-transfected DA2 cells, WT p50 csk overexpression resulted in a $\sim 50 \%$ reduction of FN-stimulated cell migration. This inhibitory effect of $\mathrm{p} 50^{\mathrm{csk}}$ was not observed when the DA2 cells were transfected with a kinase-inactive $(\mathrm{K} 222 \mathrm{M})$ mutant of $\mathrm{p} 50^{\text {csk }}$ (Fig. 5B). These results support the hypothesis that FAK Tyr397 phosphorylation and recruitment of Src-family PTKs are important components of FN-stimulated and FAK-mediated cell migration events.

To elucidate the mechanisms by which the Arg-454 or Ala712/713 FAK mutants are defective in promoting efficient cell migration, $\mathrm{FAK}^{-}$cells were transiently transfected, FNstimulated, and the various HA-tagged FAK constructs were analyzed by blotting with both anti-P.Tyr and phosphospecific antibodies directed to different FAK tyrosine phosphorylation sites (Fig. 5C). HA-blotting of the FAK IPs showed high level expression of all constructs and anti-P.Tyr blotting revealed that WT and Ala-712/713 FAK exhibited strong phosphotyrosine reactivity after FN stimulation. Both Phe-397 and Arg-454 FAK also were tyrosine phosphorylated but to a lesser extent than WT and Ala-712/713 FAK (Fig. 5C). Phospho-specific antibodies directed either to the Tyr-
577 site (pY577) within the FAK kinase domain or to the FAK autophosphorylation site at Tyr-397 (pY397) showed strong reactivity to WT and Ala-712/713 FAK, weak reactivity to Arg-454 FAK, and no reactivity to Phe-397 FAK (Fig. 5C).

Since previous studies have shown that Arg-454 FAK weakly associates with c-Src after FN-stimulation of cells (Schlaepfer and Hunter, 1996), it can be concluded that phosphorylation of Arg-454 FAK at the Tyr-397 site may be able to promote cell migration through similar types of interactions in the FAK ${ }^{-}$cells. However, Ala-712/713 FAK is strongly phosphorylated at the Tyr-397 site and within the kinase domain at Tyr-577, yet this mutant only promotes a low level of $\mathrm{FAK}^{-}$cell migration. One of the simplest interpretations of these results is that both $\mathrm{SH} 2$-mediated protein interactions at the FAK Tyr-397 site and SH3-mediated interactions at the FAK Pro-712/713 site are both required for FN-stimulated cell migration events. Mutation of either site in FAK disrupts FAK function in promoting cell motility to FN. These results support the hypothesis that FAK kinase activation after FN stimulation promotes the assembly of a multi-protein complex with FAK that is important for focal contact remodeling and cell motility events.

\section{FRNK localization to focal contacts blocks FAK- mediated cell migration events}

FRNK is an autonomously expressed protein (see Fig. 5A) in chicken embryo fibroblasts which has been shown to be a negative regulator of FAK function (Schaller et al., 1993). Exogenous expression of FRNK can inhibit both FNstimulated cell spreading in chicken embryo fibroblasts (Richardson and Parsons, 1996) and endothelial cell migration in wound healing assays (Gilmore and Romer, 1996). FRNK expression in the $\mathrm{FAK}^{-}$cells did not promote migration (Fig. 5A) and co-expression of FRNK with FAK potently inhibited FN-stimulated and FAK-mediated cell migration in a dosedependent manner (Fig. 6A). Immunoblotting whole cell lysates of the FRNK and FAK-transfected cells used in the migration assay showed that the inhibitory effects of FRNK were not due to decreases in FAK protein expression or stability (Fig. 6A).

To determine the sites important for the FRNK-mediated inhibition of cell migration, mutagenesis was performed to disrupt known protein binding sites in FRNK. Introduction of the equivalent Ala-712/713 in the first proline-rich motif of FRNK to disrupt SH3 domain-mediated interactions yielded a FRNK construct that when transfected into cells resulted in a toxic phenotype (data not shown). This result is consistent with previous observations that expression of C-terminal domain FAK constructs can generate signals promoting cell apoptosis (Ilic et al., 1998). Mutation of leucine-1034 to serine (Ser-1034) in FRNK (numbering corresponding to FAK residues) has been shown to disrupt the association of paxillin to glutathione-S-transferase (GST) fusion proteins of FRNK in vitro (Tachibana et al., 1995) and has been shown to block the focal contact localization of micro-injected GST-FRNK in fibroblasts (Tachibana et al., 1995). Introduction of Ser-1034 FRNK into $\mathrm{FAK}^{-}$or DA2 cells yielded a stable protein without toxic side effects when expressed in cells.

By transient transfection, equivalent expression levels of the FRNK or Ser-1034 FRNK were obtained in DA2 fibroblasts 
A

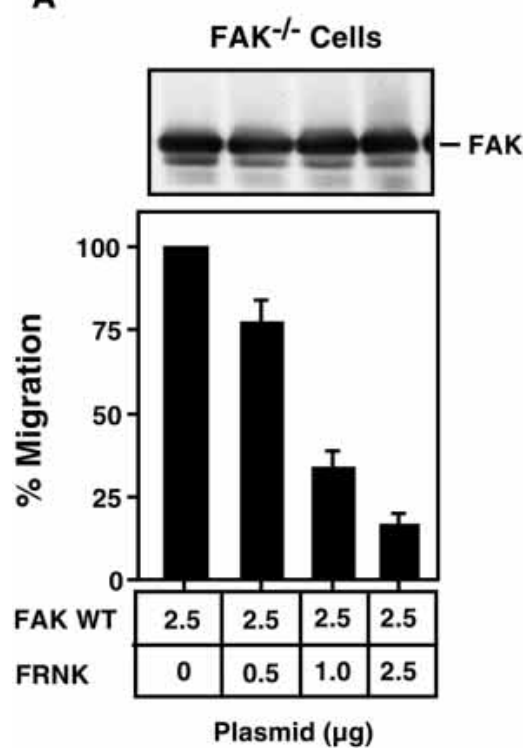

B

DA2 Haptotaxis Migration to FN

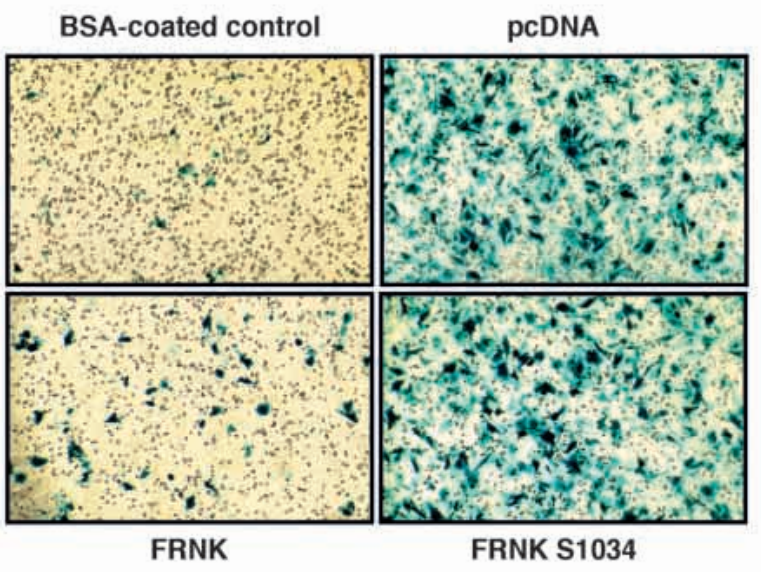

( $\beta$-gal staining of membrane underside)

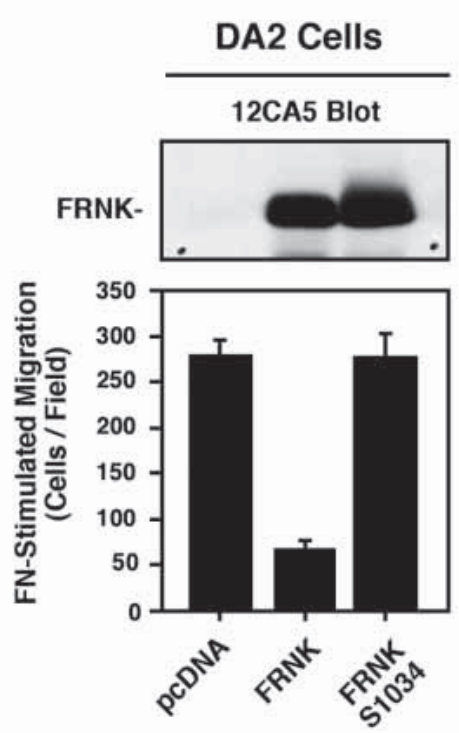

Transfected Vectors

C

$\mathrm{FAK}^{+/+}$fibroblasts

FRNK
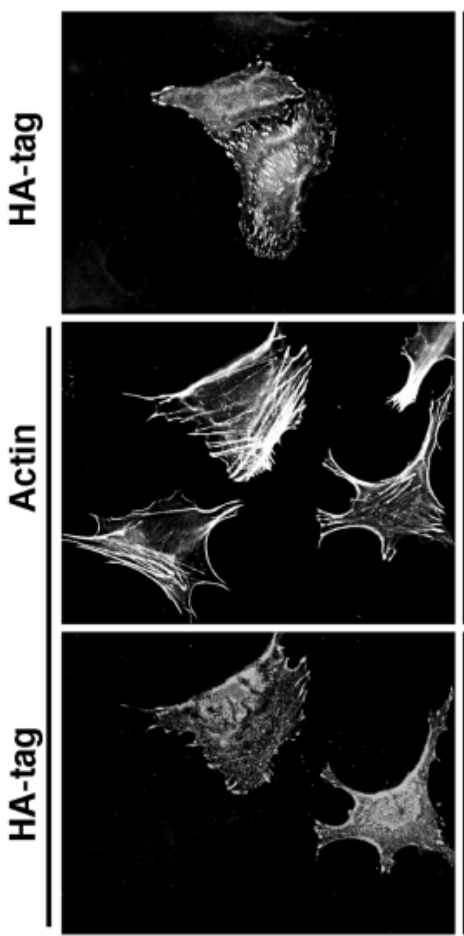

FRNK S1034
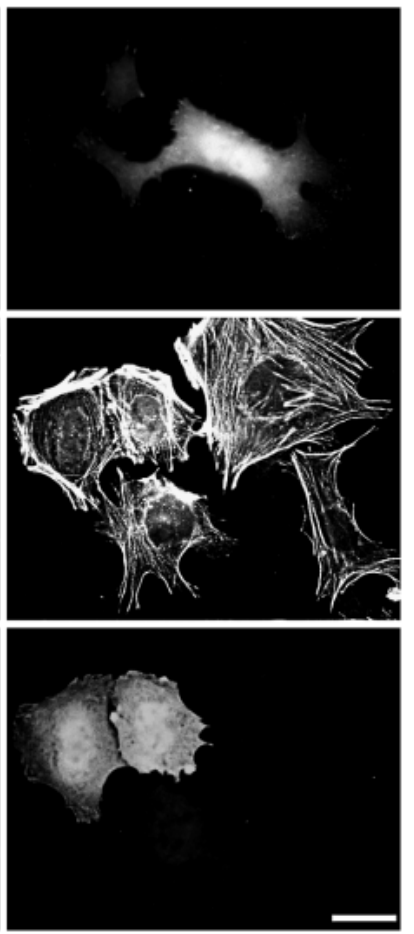

D FN-stimulated

DA2 Cells

FAK-

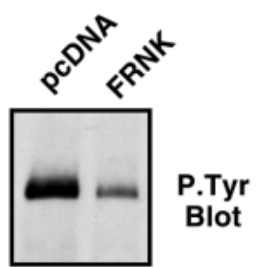

FAK-

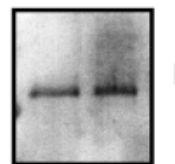

HA-tag

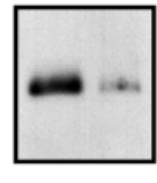

FAK

pY397

Blot

Fig. 6. FRNK expression blocks FAK-enhanced migration and tyrosine phosphorylation. (A) FAK ${ }^{-/-}$cells were transiently transfected with $\beta$ gal, the indicated amounts of FAK and FRNK plasmid DNA and analyzed in a FN haptotaxis Boyden chamber assay. FAK protein expression was verified by HA-tag (12CA5) blotting of whole cell lysates prepared from excess cells not used the migration assay. Shown is the percentage of FAK-promoted migration of the transfected $\mathrm{FAK}^{-1-}$ cells towards FN. Each bar represents the mean and standard deviation of triplicates from at least two independent experiments. (B) DA2 cells were transiently transfected with $\beta$-gal and either FRNK or Ser-1034 FRNK (S1034) followed by analysis in a FN haptotaxis Boyden chamber assay. FRNK protein expression was verified by HA-tag (12CA5) blotting of whole cell lysates prepared from excess cells not used the migration assay. Images of the X-gal stained cells on the membrane underside of the BSAcoated control or the FN-experimental points are shown. Mean values represent the number of X-gal stained cells that migrated towards FN and error bars are the standard deviation of triplicates from at least two independent experiments. (C) FRNK and Ser-1034 FRNK were detected in transiently transfected $\mathrm{FAK}^{+/+}$cells grown on FN coated coverslips by IF staining with mAb HA-tag antibody (upper panels). Transfected cells were also co-stained with phalloidin-TRITC (middle panels) and HA-tag antibodies (lower panels). Bar, $25 \mu \mathrm{m}$. (D) DA2 fibroblasts were transiently transfected with pcDNA control vector (lane 1) or FRNK (lane 2) and replated onto FN-coated dishes for 20 minutes. FAK IPs were made using affinity-purified polyclonal (\#5904) antibodies directed to the N-terminal domain of FAK. FAK IPs on the same membrane were sequentially analyzed by anti-P.Tyr blotting, anti-HA-tag blotting, followed by anti-pY397 FAK blotting. 
used in a Boyden Chamber FN haptotaxis migration assay (Fig. 6B). Similar to the dose-dependent inhibition of transient FAKmediated $\mathrm{FAK}^{-}$cell migration (Fig. 6A), transfection of FRNK into the DA2 fibroblasts potently inhibited cell migration to FN (Fig. 6B). FRNK expression did not noticeably delay either DA2 binding to FN or initial cell spreading events (data not shown). Surprisingly, high levels of Ser-1034 FRNK expression in the DA2 fibroblasts did not effect FAK-mediated cell migration (Fig. 6B). To determine the functional differences between FRNK and Ser-1034 FRNK, IF staining with antibodies to the C-terminal HA-tag antibodies was performed on transiently-transfected normal fibroblasts plated onto FN (Fig. 6C). In FRNK expressing cells, patches of strong HA-staining were detected around the cell perimeter. This staining pattern was similar to that of other focal contact associated proteins.

In contrast to the focal contact distribution of FRNK, Ser1034 FRNK staining was primarily cytoplasmic and did not strongly co-localize with focal contact sites in the $\mathrm{FAK}^{+/+}$cells (Fig. 6C). Interestingly, in cells co-stained for actin and HAtag antibodies, high levels of either FRNK or Ser-1034 FRNK did not affect the filamentous actin organization of the normal fibroblasts (Fig. 6C). Thus, while FRNK acts as a potent inhibitor of FAK-mediated fibroblast cell migration, FRNK does not act to inhibit cell binding to $\mathrm{FN}$ or act to promote changes in cytoskeletal structures.

The ability of FRNK but not Ser-1034 FRNK to inhibit FAK-mediated cell migration correlated with the strong localization of FRNK to focal contacts. Although technical limitations prevented accurate analyses to determine whether FRNK expression promoted the competitive displacement of FAK from focal contact sites, FRNK expression potently inhibited FAK tyrosine phosphorylation in FN-stimulated DA2 cells (Fig. 6D). In particular, FRNK expression prevented efficient FAK tyrosine phosphorylation at the Tyr-397 autophosphorylation/SH2 binding site (Fig. 6D, pY397). Ser1034 FRNK expression did not inhibit FAK tyrosine phosphorylation in the DA2 cells (data not shown). These results support the conclusion that the inhibitory effects of FRNK occur through the reduction of FAK tyrosine phosphorylation levels and the prevention of FAK-mediated recruitment of other signaling proteins to focal contact sites.

\section{Paxillin binding to FAK is not required for cell migration}

The Ser-1034 mutation in FRNK has been shown to disrupt both paxillin binding and focal contact localization (Tachibana et al., 1995). This point mutation was also introduced into fulllength FAK to determine its effects on FAK function in promoting cell motility. Equivalent amounts of either FAK, Ser-1034 FAK, FRNK, or Ser-1034 FRNK were isolated with antibodies to the HA-tag after transient transfection of human $293 \mathrm{~T}$ cells (Fig. 7A). Immunoblotting with antibodies to paxillin revealed that the Ser-1034 mutation disrupted paxillin association with both FAK and FRNK in vivo (Fig. 7A, lanes 2 and 4). Immunoblotting duplicate HA-tag IPs with antibodies to p130 Cas showed that both FAK and FRNK associated with equivalent amounts of p130 Cas in vivo (Fig. 7A, lanes 1 and 3). Interestingly, Ser-1034 FAK associated with a greater amount of $\mathrm{p} 130^{\mathrm{Cas}}$ compared to FAK, FRNK and Ser-1034 FRNK (Fig. 7A, lane 2). These results show that FRNK does not act to sequester large amounts of FAK-associated proteins and additionally, that the binding of paxillin and p130 Cas to FAK may be mutually exclusive.

Since the Ser-1034 mutation disrupted paxillin binding and blocked the ability of FRNK to inhibit FAK-mediated cell migration events, Ser-1034 FAK was transfected into FAK cells and was compared for its ability to promote FNstimulated cell migration in the haptotaxis Boyden chamber assay (Fig. 7B). Surprisingly, expression of Ser-1034 FAK increased $\mathrm{FAK}^{-}$cell migration $\sim 6$-fold higher than controltransfected cells. Although this level of migration was less than WT FAK, it was greater than the low level of cell migration stimulated by either of the Arg-454 or Ala-712/713 FAK constructs (see Fig. 5). Interestingly, indirect IF analyses of the transfected $\mathrm{FAK}^{-}$cells revealed that both Ser-1034 FAK and WT FAK localized to focal contact sites (Fig. 7C). In addition to the ability of Ser-1034 FAK to promote migration, FAK cells expressing high levels of Ser-1034 FAK showed distinct morphological changes, exhibiting a polarized shape characteristic of the normal and DA2 fibroblasts (Fig. 7C).

These results show that a point mutation at Leu-1034 in FRNK is sufficient to inhibit FRNK binding to paxillin and FRNK localization to focal contacts. This same mutation in FAK disrupts paxillin binding but does not block FAK localization to focal contacts or FAK function in promoting cell migration. Therefore, contrary to conclusions from previous studies (Richardson et al., 1997; Richardson and Parsons, 1996), our results do not support a role for FAK association with paxillin in promoting cell migration events. In addition, although paxillin binding may be necessary for FRNK association with focal contacts (Tachibana et al., 1995), our results with Ser-1034 FAK suggest that paxillin binding to FAK is not an absolute requirement for the focal contact localization of FAK as originally observed (Hildebrand et al., 1995). Instead, increased binding interactions of Ser-1034 FAK with other focal contact-associated proteins such as p130 Cas (Fig. 6A), talin (Chen et al., 1995), or FAK N-terminal domain interactions with $\beta$-integrin subunits (Schaller et al., 1995) may also promote FAK localization to focal contacts and allow for FAK function in enhancing cell migration.

\section{DISCUSSION}

In this study we show that the stable re-expression of epitopetagged FAK in primary FAK-null fibroblasts promoted the reversion of a rounded $\mathrm{FAK}^{-}$cell morphology to a elongated and normal fibrillar fibroblast phenotype. The effect of FAK on cell morphology was not due to a detectable redistribution of the focal contact-associated proteins vinculin, paxillin, or talin. Instead, FAK localization to focal contact sites generated signals leading to actin contractility events and the dynamic regulation of focal contact structures as observed by time course vinculin staining studies of FN-stimulated cells. The $\mathrm{FAK}^{-}$cells exhibited elevated levels of cortical actin structures, a greater number of focal contacts, and were unable to readily assume a pointed or elongated morphology upon $\mathrm{FN}$ stimulation. Contrary to initial hypotheses proposing a role for FAK in the formation of focal contacts (Richardson et al., 1997), our results confirm those from the initial knockout studies (Ilic et al., 1995) which concluded that FAK was 
involved in the regulation or turnover of focal contact structures during cell migration events.

We show in this study that stable FAK re-expression rescued the migration defects of the $\mathrm{FAK}^{-}$cells. FAK re-expression restored the ability of the $\mathrm{FAK}^{-}$cells to efficiently migrate to a FN stimulus in the absence of serum. In all studies to date, the assessment of the relative contributions of FAK in promoting cytoskeletal changes and cell migration events have been complicated by the presence of endogenous FAK. Our results showing that $\mathrm{FAK}$ re-expression in the $\mathrm{FAK}^{-}$cells at levels less than in normal fibroblasts can reverse the morphological defects in these cells and promote cell migration towards FN verifies the role of FAK as a physiological regulator of these events.

Our results with FRNK, showing the competitive inhibition of FAK-mediated cell migration events, do not support a model whereby FRNK may be acting to sequester important FAKassociated proteins. Instead, we show that FRNK localizes to focal contacts and promotes FAK dephosphorylation at the Tyr397/SH2 binding site. The Ser-1034 mutant of FRNK did not act as an inhibitor of FAK function because it did not strongly localize to focal contacts nor did its expression promote FAK
A
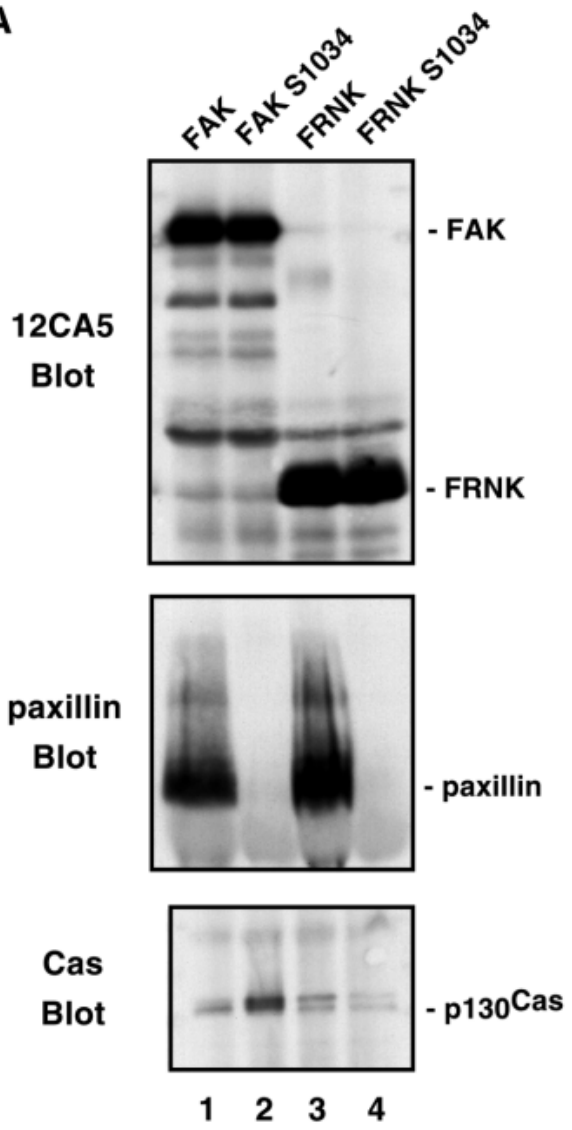

12CA5 IPs

B

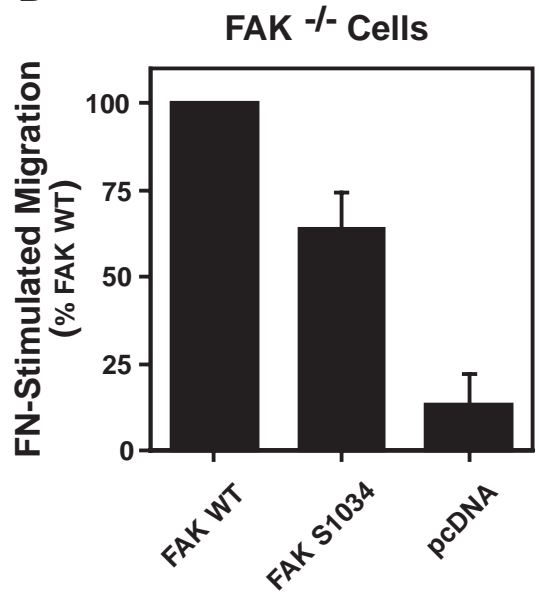

C
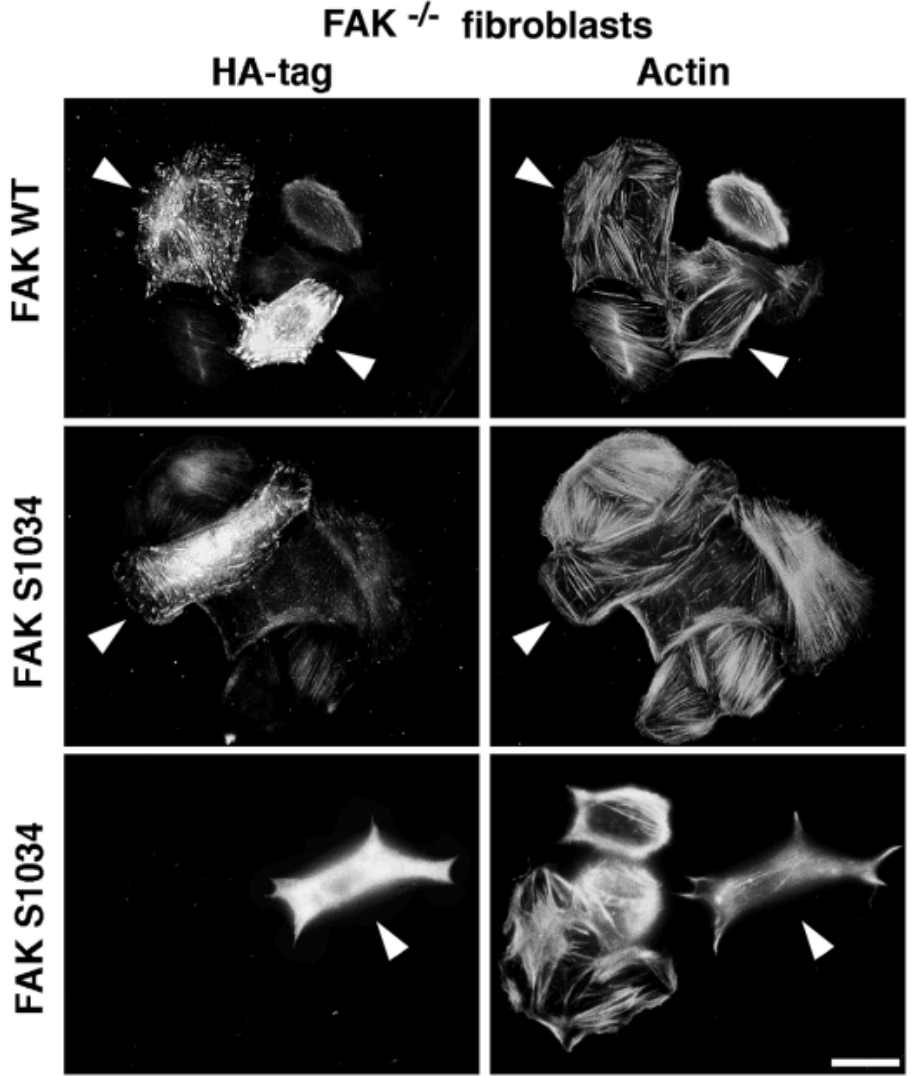

Fig. 7. Paxillin binding is not required for FAK-mediated cell migration or morphological changes. (A) $293 \mathrm{~T}$ cells were transiently transfected with either HAtagged FAK (lane 1), Ser-1034 FAK (FAK S1034, lane 2), FRNK (lane 3) or Ser1034 FRNK (FRNK S1034, lane 4) and cell lysates were made with $1 \%$ Triton lysis buffer. Equivalent expression of the FAK and FRNK constructs was verified by 12CA5 blotting of the HA-tag IPs. Membranes were sequentially analyzed by blotting with $\mathrm{mAb}$ antibodies to either paxillin or p130 Cas . (B) $\mathrm{FAK}^{-/-}$cells were transiently transfected with $\beta$-gal and either FAK, Ser-1034 FAK (FAK S1034), or pcDNA control vector and analyzed in a FN haptotaxis Boyden chamber assay. Shown is the percentage of FAK-promoted migration of the transfected cells towards FN. Each bar represents the mean and standard deviation of triplicates from at least two independent experiments. (C) HA-tagged wild-type FAK or Ser-1034 FAK (FAK S1034) were detected in transiently transfected $\mathrm{FAK}^{-1-}$ cells grown on FN coated coverslips by IF staining with HA-tag antibodies (left panels) and phalloidin-TRITC (right panels). Transfected cells are indicated by arrowheads. Bar, $25 \mu \mathrm{m}$. 
dephosphorylation. Interestingly, Ser-1034 full-length FAK both localized to focal contacts and promoted cell migration supporting the hypothesis that FAK functions at these sites to initiate cell migration. The fact that Ser-1034 FAK did not detectably associate with paxillin suggests that this interaction is not required for focal contact localization or FAK-mediated cell migration events. Instead, as depicted in the summary model (Fig. 8), FAK localization to sites of FN receptor integrin clustering is essential for the recruitment of other target proteins required to transduce signals for integrinstimulated cell migration events.

\section{Model of FAK function in promoting cell migration}

Expression of various FAK mutants in the FAK ${ }^{-}$cells showed that FAK kinase activity, the Tyr-397/SH2 domain binding site, and the first proline-rich SH3 binding region in the FAK Cterminal domain were individually needed to promote $\mathrm{FAK}^{-}$ cell migration to $\mathrm{FN}$ whereas direct paxillin binding to FAK was not required. The exact mechanism as to how FAK functions to promote cell shape changes and which signaling pathway(s) it uses to promote haptotaxis cell migration will require further analyses. However, the fact that the individual FAK Phe-397, Arg-454, or Ala-712/713 point mutations prevented full FAK function in promoting cell migration suggests that FNstimulated increases in FAK kinase activity lead to the recruitment of both $\mathrm{SH} 2$ and $\mathrm{SH} 3$ domain-containing signaling proteins and that a multi-protein complex with FAK is required for efficient cell migration events (Fig. 8).

One important finding in our studies was that FAK kinase activity was required for efficient FN-stimulated cell migration events. This is significant since FAK kinase activity is activated by FN stimulation of cells and gene knockout studies have suggested that FAK is a physiological mediator of both FN and $\beta 1$ integrin-generated signals (Ilic et al., 1995). Previous studies have shown that overexpression of kinase-inactive FAK in $\mathrm{CHO}$ cells functions equally as well as WT FAK in enhancing cell migration (Cary et al., 1996). In this study, we show that kinase-inactive (Arg-454) FAK is weakly tyrosine phosphorylated at Tyr-397 and previous studies have shown that this mutant is weakly associated with Src-family PTKs after FN stimulation of 293T cells (Schlaepfer and Hunter, 1996). Whereas in CHO cells, this presumed adaptor protein function of Arg-454 FAK may be sufficient to promote cell migration (Cary et al., 1996), our studies show that FAK kinase activity is required for maximal Tyr-397 site phosphorylation and full FAK function in promoting $\mathrm{FAK}^{-}$fibroblast migration.

Although elevated levels of FAK kinase activity can directly phosphorylate associated signaling proteins such as p130 Cas (Tachibana et al., 1997) and Shc (Schlaepfer et al., 1998), one of the main requirements of FAK kinase activity may be to autophosphorylate the FAK Tyr-397 site and to promote the recruitment of $\mathrm{SH} 2$ domain-containing signaling proteins to focal contact sites (Fig. 7). This multi-functional phosphorylation site on FAK is the binding site for the SH2 domains of Src-family PTKs, the p85 subunit of PI 3-kinase, and the Shc adaptor protein. Both PI 3-kinase activation (Keely et al., 1997) and signals downstream of Shc tyrosine phosphorylation leading to ERK2/MAP kinase activation (Klemke et al., 1997) have been shown to be important components of integrin-stimulated cell migration events. Inhibitors of the ERK2/MAP kinase cascade can block FN-

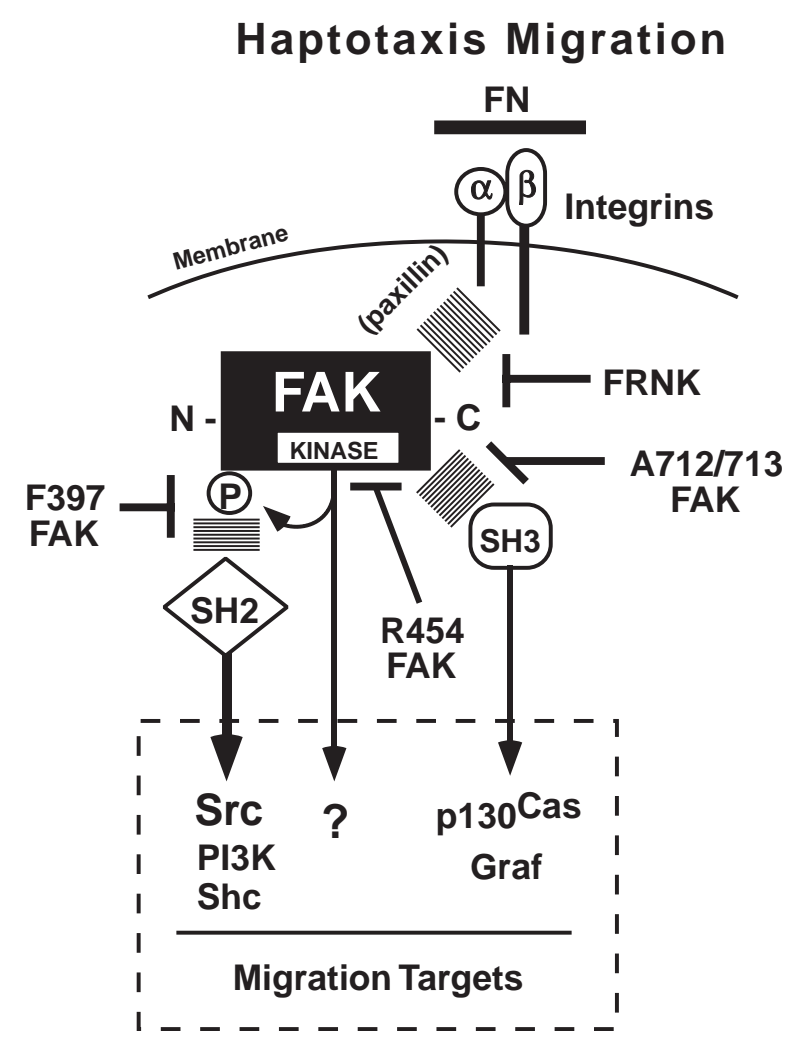

Fig. 8. Model of FAK function in promoting integrin-stimulated cell migration. Fibronectin binding to transmembrane integrin receptors leads to the activation of FAK tyrosine kinase activity. Subsequently, Tyr-397 in FAK becomes phosphorylated by an autophosphorylation mechanism initiating the recruitment of $\mathrm{SH} 2$ domain-containing proteins such as Src family PTKs, the p85 subunit of PI3K and Shc. In addition, FAK serves as a scaffold for $\mathrm{SH} 3$ domain-containing proteins like $\mathrm{p} 130^{\mathrm{Cas}}$ or Graf which bind to either of two C-terminal proline-rich regions. FAK localization to focal contact sites and regions of integrin clustering occurs independent of paxillin binding to FAK. However, FAK activation by FN stimulation of cells can be blocked by overexpression of the C-terminal domain of FAK termed FRNK which contains the paxillin binding domain. FAK functions to promote $\mathrm{FN}$-stimulated haptotaxis cell migration through the recruitment of other signaling proteins and by promoting changes in actin cytoskeletal structures leading to cell elongation. Mutations at the FAK autophosphorylation site (F397), in the kinase domain (R454), and at the first proline-rich motif (A712/713) impede the formation of a multi-protein signaling complex and thereby inhibit FAK function in promoting cell migration.

stimulated fibroblast cell migration (Anand-Apte et al., 1997) and this connection may be at the level of myosin light chain activation (Klemke et al., 1997). Interestingly, both transient Pyk2 overexpression and FAK re-expression in the $\mathrm{FAK}^{-}$cells potentiated FN-stimulated ERK2 activation; however Pyk2 did not efficiently promote $\mathrm{FAK}^{-}$cell migration to $\mathrm{FN}$ compared to FAK (Sieg et al., 1998). Since Pyk2 exhibits a peri-nuclear localization in the $\mathrm{FAK}^{-}$cells, it may be that the differential localization of signaling complexes with FAK to focal contact sites is an important factor for cell motility or that FNstimulated ERK2 activation is not the rate-limiting step preventing $\mathrm{FAK}^{-}$cell migration.

Our studies showing that $\mathrm{p} 50^{\mathrm{csk}}$ overexpression could inhibit 
FN-stimulated and FAK-mediated cell migration supports the conclusion that the recruitment of active Src-family PTKs to focal contact sites is an important factor promoting cell motility events. Indeed, it has been proposed that the FAKmediated recruitment of active Src-family PTKs to focal contact sites leads to the disassembly of cell-substratum adhesion sites (Fincham and Frame, 1998) and it has been shown that overexpression of active c-Src can reverse the negative effects of FRNK on chicken embryo fibroblast cell spreading (Richardson et al., 1997). These results are also supported by the studies performed with triple Src-family PTK-null ( $\mathrm{Src}^{-/-}, \mathrm{Yes}^{-/-}, \mathrm{Fyn}^{-/-}$) fibroblasts (SYF cells) (Klinghoffer et al., 1999). In these cells, both FN-stimulated tyrosine phosphorylation events and haptotaxis cell migration to FN were dramatically reduced compared to SYF cells reexpressing c-Src.

Although it is unclear how this Src-FAK complex functions to promote cell migration, our results showing reduced levels of cell migration with the Ala-712/713 FAK mutant also suggest that other $\mathrm{SH} 3$-mediated protein binding interactions with FAK are also required for FN-stimulated cell motility events. The fact that Ala-712/713 FAK exhibited high levels of FN-stimulated phosphorylation at the Tyr-397 site but only promoted low levels of cell migration suggest that multiple protein binding interactions with FAK function to coordinate cell migration events. We speculate that FN-stimulated increases in FAK kinase activity promote the recruitment of Src-family PTKs followed by the sequential (Src-mediated) phosphorylation of target proteins bound to the FAK Pro712/713 site.

One common phosphorylation target of both FAK and Src kinase activities is the adaptor protein, p130 Cas . Interestingly, p130 Cas-l- fibroblasts also exhibit defects in the assembly of actin filaments in the formation of actin stress fibers as do the FAK $^{-}$cells (Honda et al., 1998). The Ala-712/713 mutation in FAK disrupts the primary p130 ${ }^{\text {Cas }} \mathrm{SH} 3$ domain binding site (Polte and Hanks, 1995, 1997) and also severely limits the ability of this FAK mutant to promote $\mathrm{FAK}^{-}$cell migration. Previous studies have shown that overexpression of an Ala712/715 FAK mutant in CHO cells did not promote elevated cell migration and that co-expression of the $\mathrm{SH} 3$ domain of p130 Cas can inhibit FAK-enhanced CHO cell motility (Cary et al., 1998). Our findings that Ser-1034 FAK associated with greater amounts of $\mathrm{p} 130^{\mathrm{Cas}}$ by co-immunoprecipitation may reflect the pathway through which this FAK mutant promotes cell migration. It has been proposed that $\mathrm{Crk} \mathrm{SH} 2$ domainmediated adaptor protein binding to tyrosine-phosphorylated p130 ${ }^{\text {Cas }}$ is important for Rac-dependent and Ras-independent cell migration events (Klemke et al., 1998). However, since other adaptor proteins such as Nck bind to p130 Cas (Schlaepfer et al., 1997), it is possible that multiple pathways downstream of $130^{\text {Cas }}$ are important for promoting cell migration events.

Another target protein that may be involved in FAKmediated cell migration events is the Rho GTPase-activating protein, Graf (Hildebrand et al., 1996). Graf binds to FAK through SH3 domain-mediated interactions and overexpression of Graf in fibroblasts affects actin stress fiber formation and cell morphology (Taylor et al., 1999). This connection is interesting since FAK re-expression in the $\mathrm{FAK}^{-}$cells results in the dynamic rearrangement of cortical and filamentous actin structures. Recent studies have shown that Rho activity is transiently inhibited during the initial period of FN-stimulated cell spreading (Ren et al., 1999) and this transient inactivation of Rho occurs during the same time course of maximal FAK kinase activation (Schlaepfer et al., 1998).

In summary, one of the best analogies to describe the role of FAK in cells is that it functions as an integrin-activated scaffold for the proper localization and assembly of signaling complexes through the coordinated recruitment of Src-family PTKs, GTPase regulatory proteins, and modular adaptor proteins. Future efforts to determine and decipher these FAKmediated interactions will continue to enhance our knowledge on the regulatory mechanisms governing cell growth, shape, and migration.

We thank K. C. Jones for assistance in isolating FAK re-expressing clones, Shannon Reider for technical support, and Dusko Ilic for expertise in IF analyses and critical review of the manuscript. We appreciate the generosity of Erik Schaefer for the phosphotyrosinespecific antibodies to FAK and we also thank David Cheresh for access to an epifluorescence-equipped microscope. This work was supported by Public Health Services grant R29 CA75240 (D.D.S.) from the National Cancer Institute, support from the American Cancer Society (RPG-98-109-01-TBE), and a Grant-In-Aid from the American Heart Association (9750682N) to D.D.S. D.J.S. was supported by NRSA postdoctoral Training Grant (HL07195-21) and C.R.H. was support by a postdoctoral fellowship from the Deutsche Forschungsgemeinschaft (Ha 2856/1-1). This is manuscript \#12384IMM from The Scripps Research Institute.

\section{REFERENCES}

Anand-Apte, B., Zetter, B. R., Viswanathan, A., Qiu, R. G., Chen, J., Ruggieri, R. and Symons, M. (1997). Platelet-derived growth factor and fibronectin-stimulated migration are differentially regulated by the Rac and extracellular signal-regulated kinase pathways. J. Biol. Chem. 272, 3068830692.

Angers-Loustau, A., Cote, J.-F., Charest, A., Dowbenko, D., Spencer, S., Lasky, L. A. and Tremblay, M. L. (1999). Protein tyrosine phosphatasePEST regulates focal adhesion disassembly, migration, and cytokinesis in fibroblasts. J. Cell Biol. 144, 1019-1031.

Calalb, M., Polte, T. and Hanks, S. K. (1995). Tyrosine phosphorylation of focal adhesion kinase at sites in the catalytic domain regulates kinase activity: a role for the Src family kinases. Mol. Cell. Biol. 15, 954-963.

Cary, L. A., Chang, J. F. and Guan, J.-L. (1996). Stimulation of cell migration by overexpression of focal adhesion kinase and its association with Src and Fyn. J. Cell Sci. 108, 1787-1794.

Cary, L. A., Han, D. C., Polte, T. R., Hanks, S. K. and Guan, J.-L. (1998). Identification of $\mathrm{p} 130^{\mathrm{Cas}}$ as a mediator of focal adhesion kinase-promoted cell migration. J. Cell Biol. 140, 211-221.

Chen, H. C., Appeddu, P. A., Isoda, H. and Guan, J. L. (1996). Phosphorylation of tyrosine 397 in focal adhesion kinase is required for binding phosphatidylinositol 3-kinase. J. Biol. Chem. 271, 26329-26334.

Chen, H. C., Appeddu, P. A., Parsons, J. T., Hildebrand, J. D., Schaller, M. D. and Guan, J. L. (1995). Interaction of focal adhesion kinase with cytoskeletal protein talin. J. Biol. Chem. 270, 16995-16999.

Fincham, V. J. and Frame, M. C. (1998). The catalytic activity of Src is dispensable for translocation to focal adhesions but controls the turnover of these structures during cell motility. EMBO J. 17, 81-92.

Frisch, S. M., Vuori, K., Ruoslahti, E. and Chan-Hui, P. Y. (1996). Control of adhesion-dependent cell survival by focal adhesion kinase. J. Cell Biol. 134, 793-799.

Furuta, Y., Ilic, D., Kanazawa, S., Takeda, N., Yamamoto, T. and Aizawa, S. (1995). Mesodermal defect in late phase of gastrulation by a targeted mutation of focal adhesion kinase, FAK. Oncogene 11, 1989-1995.

George, E. L., Georges-Labouesse, E. N., Patel-King, R. S., Rayburn, H. and Hynes, R. O. (1993). Defects in mesoderm, neural tube and vascular development in mouse embryos lacking fibronectin. Development 119, 1079-1091. 
Gilmore, A. P. and Romer, L. H. (1996). Inhibition of focal adhesion kinase (FAK) signaling in focal adhesions decreases cell motility and proliferation. Mol. Biol. Cell 7, 1209-1224.

Hanks, S. K. and Polte, T. R. (1997). Signaling through focal adhesion kinase. BioEssays 19, 137-145.

Hildebrand, J. D., Schaller, M. D. and Parsons, J. T. (1995). Paxillin, a tyrosine phosphorylated focal adhesion-associated protein binds to the carboxyl terminal domain of focal adhesion kinase. Mol. Biol. Cell 6, 637647.

Hildebrand, J. D., Taylor, J. M. and Parsons, J. T. (1996). An SH3 domaincontaining GTPase-activating protein for Rho and Cdc42 associates with focal adhesion kinase. Mol. Cell. Biol. 16, 3169-3178.

Honda, H., Oda, H., Nakamoto, T., Honda, Z., Sakai, R., Suzuki, T., Saito, T., Nakamura, K., Nakao, K., Ishikawa, T. et al. (1998). Cardiovascular anomaly, impaired actin bundling and resistance to Src-induced transformation in mice lacking p130Cas. Nature Genet. 19, 361-365.

Ilic, D., Furuta, Y., Kanazawa, S., Takeda, N., Sobue, K., Nakatsuji, N., Nomura, S., Fujimoto, J., Okada, M., Yamamoto, T. et al. (1995). Reduced cell motility and enhanced focal adhesion contact formation in cells from FAK-deficient mice. Nature 377, 539-544.

Ilic, D., Almeida, E. A., Schlaepfer, D. D., Dazin, P., Aizawa, S. and Damsky, C. H. (1998). Extracellular matrix survival signals transduced by focal adheison kinase suppress p53-mediated apoptosis. J. Cell Biol. 143, 547-560.

Keely, P. J., Westwick, J. K., Whitehead, I. P., Der, C. J. and Parise, L. V. (1997). Cdc42 and Rac1 induce integrin-mediated cell motility and invasiveness through PI(3)K. Nature 390, 632-636.

Klemke, R. L., Cai, S., Giannini, A. L., Gallagher, P. J., de Lanerolle, P. and Cheresh, D. A. (1997). Regulation of cell motility by mitogen-activated protein kinase. J. Cell Biol. 137, 481-492.

Klemke, R. L., Leng, J., Molander, R., Brooks, P. C., Vuori, K. and Cheresh, D. A. (1998). Cas/Crk coupling serves as a 'molecular switch' for induction of cell migration. J. Cell Biol. 140, 961-972.

Klinghoffer, R. A., Sachsenmaier, C., Cooper, J. A. and Soriano, P. (1999), Src family kinases are required for integrin but not PDGFR signal transduction. EMBO J. 18, 2459-2471.

Owens, L. V., Xu, L. H., Craven, R. J., Dent, G. A., Weiner, T. M., Kornberg, L., Liu, E. T. and Cance, W. G. (1995). Overexpression of the focal adhesion kinase ( $125^{\mathrm{FAK}}$ ) in invasive human tumors. Cancer Res. $\mathbf{5 5}$, 2752-2755.

Polte, T. R. and Hanks, S. K. (1995). Interaction between focal adhesion kinase and Crk-associated tyrosine kinase substrate p130 Cas. Proc. Nat. Acad. Sci. USA 92, 10678-10682.

Polte, T. R. and Hanks, S. K. (1997). Complexes of focal adhesion kinase (FAK) and Crk-associated substrate (p130 Cas) are elevated in cytoskeletonassociated fractions following adhesion and Src transformation. Requirements for Src kinase activity and FAK proline-rich motifs. J. Biol. Chem. 272, 5501-5509.

Ren, X. D., Kiosses, W. B. and Schwartz, M. A. (1999). Regulation of the small GTP-binding protein Rho by cell adhesion and the cytoskeleton. EMBO J. 18, 578-585.

Richardson, A. and Parsons, J. T. (1996). A mechanism for regulation of the adhesion-associated protein tyrosine kinase $\mathrm{pp} 125^{\mathrm{FAK}}$. Nature 380, 538-540.

Richardson, A., Malik, R. K., Hildebrand, J. D. and Parsons, J. T. (1997). Inhibition of cell spreading by expression of the C-terminal domain of focal adhesion kinase (FAK) is rescued by coexpression of Src or catalytically inactive FAK: a role for paxillin tyrosine phosphorylation. Mol. Cell Biol. 17, 6906-6914.

Schaller, M. D., Borgman, C. A. and Parsons, J. T. (1993). Autonomous expression of a noncatalytic domain of the focal adhesion-associated protein tyrosine kinase pp125 ${ }^{\mathrm{FAK}}$. Mol. Cell. Biol. 13, 785-791.
Schaller, M. D., Otey, C. A., Hildebrand, J. D. and Parsons, J. T. (1995). Focal adhesion kinase and paxillin bind peptides mimicking $\beta$ integrin cytoplasmic domains. J. Cell Biol. 130, 1181-1187.

Schlaepfer, D. D., Hanks, S. K., Hunter, T. and van der Geer, P. (1994). Integrin-mediated signal transduction linked to Ras pathway by Grb2 binding to focal adhesion kinase. Nature 372, 786-791.

Schlaepfer, D. D. and Hunter, T. (1996). Evidence for in vivo phosphorylation of the Grb2 SH2-domain binding site on focal adhesion kinase by Src-family protein-tyrosine kinases. Mol. Cell. Biol. 16, 56235633.

Schlaepfer, D. D. and Hunter, T. (1997). FAK overexpression enhances Rasdependent integrin signaling to ERK2/mitogen-activated protein kinase through interactions with and activation of c-Src. J. Biol. Chem. 272, 1318913195.

Schlaepfer, D. D., Broome, M. A. and Hunter, T. (1997). Fibronectinstimulated signaling from a focal adhesion kinase-c-Src complex: involvement of the Grb2, p130 Cas , and Nck adaptor proteins. Mol. Cell. Biol. 17, 1702-1713.

Schlaepfer, D. D. and Hunter, T. (1998). Integrin signaling and tyrosine phosphorylation: Just the FAKs? Trends Cell Biol. 8, 151-157.

Schlaepfer, D. D., Jones, K. C. and Hunter, T. (1998). Multiple Grb2mediated integrin-stimulated signaling pathways to ERK2/mitogenactivated protein kinase: Summation of both $\mathrm{c}-\mathrm{Src}$ and FAK-initiated tyrosine phosphorylation events. Mol. Cell. Biol. 18, 2571-2585.

Schlaepfer, D. D., Hauck, C. R. and Sieg, D. J. (1999). Signaling through focal adhesion kinase. Prog. Biophys. Mol. Biol. 71, 435-478.

Sieg, D. J., Ilic, D., Jones, K. C., Damsky, C. H., Hunter, T. and Schlaepfer, D. D. (1998). Pyk2 and Src-family protein-tyrosine kinases compensate for the loss of FAK in fibronectin-stimulated signaling events but Pyk2 does not fully function to enhance $\mathrm{FAK}^{-}$cell migration. $E M B O$ J. 17, 5933-5947.

Stephens, L. E., Sutherland, A. E., Klimanskaya, I. V., Andrieux, A., Meneses, J., Pedersen, R. A. and Damsky, C. H. (1995). Deletion of $\beta 1$ integrins in mice results in inner cell mass failure and peri-implantation lethality. Genes Dev. 9, 1883-1895.

Tachibana, K., Sato, T., D’Avirro, N. and Morimoto, C. (1995). Direct association of pp125 $\mathrm{FAK}$ with paxillin, the focal adhesion-targeting mechanism of pp125 $5^{\mathrm{FAK}}$. J. Exp. Med. 182, 1089-1100.

Tachibana, K., Urano, T., Fujita, H., Ohashi, Y., Kamiguchi, K., Iwata, S., Hirai, H. and Morimoto, C. (1997). Tyrosine phosphorylation of Crkassociated substrates by focal adhesion kinase. A putative mechanism for the integrin-mediated tyrosine phosphorylation of Crk-associated substrates. J. Biol. Chem. 272, 29083-29090.

Tamura, M., Gu, J., Matsumoto, K., Aota, S., Parsons, R. and Yamada, K. M. (1998). Inhibition of cell migration, spreading, and focal adhesions by tumor suppressor PTEN. Science 280, 1614-1617.

Tamura, M., Gu, J., Takino, T. and Yamada, K. M. (1999). Tumor suppressor PTEN inhibition of cell invasion, migration, and growth differential involvement of focal adhesion kinase and $130^{\text {Cas }}$. Cancer Res 59, 442-449.

Taylor, J. M., Macklem, M. M. and Parsons, J. T. (1999). Cytoskeletal changes induced by GRAF, the GTPase regulator associated with focal adhesion kinase, are mediated by Rho. J. Cell Sci. 112, 231-242.

Vuori, K., Hirai, H., Aizawa, S. and Ruoslahti, E. (1996). Induction of p130 ${ }^{\mathrm{Cas}}$ signaling complex formation upon integrin-mediated cell adhesion: a role for the Src family kinases. Mol. Cell. Biol. 16, 2606-2613.

Yu, D. H., Qu, C. K., Henegariu, O., Lu, X. and Feng, G. S. (1998). Proteintyrosine phosphatase Shp-2 regulates cell spreading, migration, and focal adhesion. J. Biol. Chem. 273, 21125-21131.

Zhao, J.-H., Reiske, H. and Guan, J.-L. (1998). Regulation of the cell cycle by focal adhesion kinase. J. Cell Biol. 143, 1997-2008. 